\title{
Preprocessing of gravity gradients at the GOCE high-level processing facility
}

\author{
Johannes Bouman - Sietse Rispens - Thomas Gruber • \\ Radboud Koop • Ernst Schrama • Pieter Visser • \\ Carl Christian Tscherning • Martin Veicherts
}

Received: 3 March 2008 / Accepted: 2 October 2008 / Published online: 30 October 2008

(C) The Author(s) 2008. This article is published with open access at Springerlink.com

\begin{abstract}
One of the products derived from the gravity field and steady-state ocean circulation explorer (GOCE) observations are the gravity gradients. These gravity gradients are provided in the gradiometer reference frame (GRF) and are calibrated in-flight using satellite shaking and star sensor data. To use these gravity gradients for application in Earth scienes and gravity field analysis, additional preprocessing needs to be done, including corrections for temporal gravity field signals to isolate the static gravity field part, screening for outliers, calibration by comparison with existing external gravity field information and error assessment. The temporal gravity gradient corrections consist of tidal and nontidal corrections. These are all generally below the gravity gradient error level, which is predicted to show a $1 / f$ behaviour
\end{abstract}

J. Bouman · S. Rispens

SRON Netherlands Institute for Space Research,

Utrecht, The Netherlands

J. Bouman $\cdot$ E. Schrama $\cdot$ P. Visser

Delft Institute of Earth Observation and Space Systems (DEOS),

Faculty of Aerospace Engineering, Delft University of Technology,

Delft, The Netherlands

Present Address:

J. Bouman $(\bowtie)$

Deutsches Geodätisches Forschungsinstitut (DGFI),

Munich, Germany

e-mail: bouman@dgfi.badw.de

T. Gruber

Institut für Astronomische und Physikalische Geodäsie (IAPG),

Technische Universität München, Munich, Germany

R. Koop

Ambonstraat 1, 2612 BL, Delft, The Netherlands

C. C. Tscherning $\cdot$ M. Veicherts

Niels Bohr Institute, University of Copenhagen,

Copenhagen, Denmark for low frequencies. In the outlier detection, the $1 / f$ error is compensated for by subtracting a local median from the data, while the data error is assessed using the median absolute deviation. The local median acts as a high-pass filter and it is robust as is the median absolute deviation. Three different methods have been implemented for the calibration of the gravity gradients. All three methods use a high-pass filter to compensate for the $1 / f$ gravity gradient error. The baseline method uses state-of-the-art global gravity field models and the most accurate results are obtained if star sensor misalignments are estimated along with the calibration parameters. A second calibration method uses GOCE GPS data to estimate a low-degree gravity field model as well as gravity gradient scale factors. Both methods allow to estimate gravity gradient scale factors down to the $10^{-3}$ level. The third calibration method uses high accurate terrestrial gravity data in selected regions to validate the gravity gradient scale factors, focussing on the measurement band. Gravity gradient scale factors may be estimated down to the $10^{-2}$ level with this method.

Keywords GOCE · High-level processing facility · Gravity gradients $\cdot$ Preprocessing $\cdot$ Calibration

\section{Introduction}

The gravity field and steady-state ocean circulation explorer (GOCE) is the European Space Agency's (ESA) first core explorer mission in the Living Planet programme to be launched in 2008. The aim is to determine the static gravity field of the Earth from space with unprecedented accuracy and resolution. To this end, GOCE will carry an electrostatic gravity gradiometer, as well as a GPS instrument to allow highlow satellite-to-satellite tracking (SST), and star cameras 
(ESA 1999; Gruber and Rummel 2006). The scientific processing of the GOCE data, known as Level $1 b$ to Level 2 processing, will be done by the high-level processing facility (HPF), which is part of the GOCE Ground Segment (see e.g. Koop et al. 2007).

The Level 1b gravity gradients (GGs) are derived from the gradiometer observations which have been calibrated inflight using satellite shaking and star sensor data (Cesare and Catastini 2005). One of the tasks of the GOCE HPF is to preprocess the Level 1b GGs, that is, they will be corrected for temporal gravity field variations, outliers are searched for and flagged if detected, and the GGs will be externally calibrated and their error will be assessed. The Level 2 GGs thus obtained are one of the key observations, together with precise science orbits, from which other Level 2 products, such as global gravity field models, in terms of spherical harmonic coefficients, gridded geoid heights and gravity anomalies, will be derived. In addition, the Level 2 gradients themselves may be directly used in Earth sciences, typically for local and regional applications focusing on smaller spatial scales (see e.g. Eshagh and Sjöberg 2008; Li 2001; Pawlowski 1998).

The GOCE gradiometer has been designed such that the GG errors are minimal in the measurement band (MB) between $5 \mathrm{mHz}$ and $0.1 \mathrm{~Hz}$, corresponding to the medium to high wavelength gravity field features that are to be resolved from this novel instrument on board of GOCE. Below the MB, the GG error power spectral density (PSD) is expected to show $1 / f$ behaviour (ESA 1999). Long-wavelength gravity field information is to be extracted from the SST observations, which thus complement the GGs. In this paper, we present the GOCE gravity gradient preprocessing algorithms as they are implemented in the HPF. Partially, these algorithms have been presented earlier (Bouman and Koop 2003b; Bouman et al. 2004; Bouman 2004), but a complete presentation of the preprocessing has not been given so far.

In the outlier detection as well as in the external calibration, global gravity field models are used to compute model GGs. These are used for comparison with the GOCE GGs. A challenge in this comparison is the $1 / f$ behaviour of the GOCE GG errors for low frequencies. As an example, see Fig. 1, where the time series of 1 day are plotted for the measured $V_{X X}$ and the error free $V_{X X}\left(V_{X X}\right.$ is the second spatial derivative of the gravitational potential in the $X$-direction with, in this example, the $X$-axis approximately along the satellite track). Clearly a large, slowly varying error is superimposed on the true GGs. The PSD of this error as well as of the true signal are plotted in Fig. 2. The signal has most of its power at one and two cycles per revolution (CPR) due to the central term and flattening (J2) of the Earth's gravity field, and the signal power is above the error power for these frequencies. The error starts to dominate the signal for frequencies below one CPR. To reduce the effect of the $1 / f$ error, one could high-pass filter the gravity gradients. However, such a

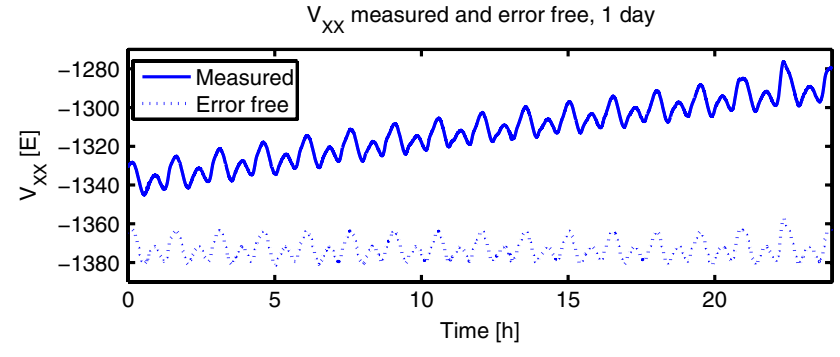

Fig. 1 Time series of 1 day of measured and error-free $V_{X X}$ GG

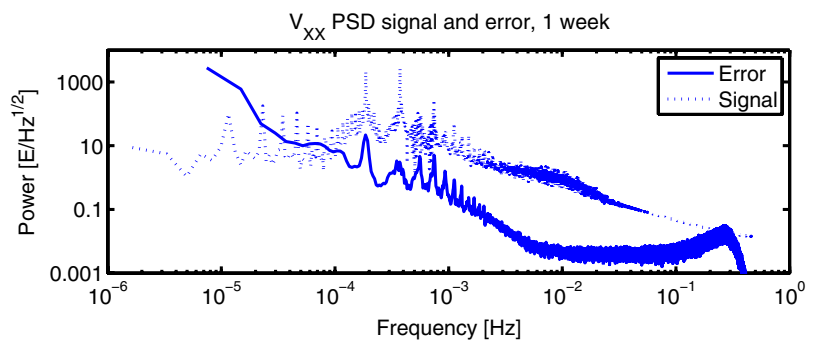

Fig. 2 PSD of 1 week of $V_{X X}$ errors and error-free $V_{X X}$ signal. Signal is large at one and two CPR $\left(\approx 2 \times 10^{-4}\right.$ and $\left.4 \times 10^{-4} \mathrm{~Hz}\right)$

filter may redistribute the signal power of outliers, if they are present, to adjacent observations, which is undesirable. We will discuss how the long wavelength error is dealt with in the outlier detection. Moreover, we will present a method that automatically finds the optimal cut-off frequency for the high-pass filter that is applied in the external calibration using global gravity field models. The method balances the tradeoff between reducing the $1 / f$ error and keeping as much as possible the signal power for low frequencies.

Furthermore, in the original design, the GOCE GG would be given in the local orbital reference frame (LORF) (ESA 1999), which is determined by the GOCE SST data. The LORF is defined with the $X$-axis along track in the direction of the velocity vector, the $Z$-axis perpendicular to this axis in the orbital plane (close to the radial direction) and the $Y$-axis cross-track completing a right-handed frame. It became, however, evident that it will be necessary to use the magnetic torquers to keep the satellite and the gradiometer as good as possible aligned with the LORF, resulting in yaw, roll and pitch angles of a few degrees (see e.g. Pail 2004; Visser 2007). The gradiometer reference frame (GRF) in which the GGs are given does therefore no longer coincide with the LORF. As a result, a combination of GOCE star tracker and/or gradiometer data has to be used to determine the GRF. Two associated problems are that on the one hand our knowledge of the GRF may suffer from star sensor or gradiometer misalignments, while on the other hand the use of gradiometer quaternions asks for a clear description of what we see as external in external calibration. We will discuss our external calibration methods and how they are related to each other. Specifically, we will explain how the external calibration method using terrestrial gravity data has evolved 
with respect to the original method that used least-squares collocation (Arabelos and Tscherning 1998; Bouman et al. 2004; Tscherning et al. 2006). In addition, we will explain how GRF misalignments may be dealt with in the external calibration method that uses global gravity field models.

Section 2 outlines the GG preprocessing scheme, while Sects. 3-5 deal with temporal gravity field corrections, outlier detection and external calibration, respectively. The methods are discussed and illustrated using simulated GOCE data. Section 6 contains the conclusions.

\section{Gravity gradient preprocessing}

Because the main goal of the GOCE mission is to provide a model of the Earth's static gravity field (ESA 1999), the GOCE gravity gradients need to be corrected for temporal gravity field variations such as solid-Earth and ocean tides, third bodies (Sun, Moon), pole tide, and oceanic and atmospheric mass redistributions. Also outliers that may occur in the GOCE gravity gradients need to be searched for and detected in the preprocessing step (Bouman 2004). Along with the external calibration of the observations (Bouman et al. 2004), the error of the observations needs to be assessed. For the latter purpose, one could use the GOCE data themselves and perform an internal assessment (see e.g. Albertella et al. 2000; Jarecki et al. 2006; Jarecki and Müller 2007). Bouman and Koop (2003b) used along-track interpolation of GGs, which seems to be an adequate tool for error assessment in the MB. The steps for GG preprocessing are (1) correction for temporal gravity field variations; (2) outlier detection and flagging; (3) external calibration and error assessment.

These preprocessing steps will lead to corrected and calibrated Level 2 GGs in the GRF, which are one of the GOCE final products. These GGs are also input to the gravity field analysis as well as to the frame transformation, which computes GGs in a local north-oriented frame (LNOF). In contrast to the GRF, the LNOF is directly related to the Earth (see Gruber et al. 2007a,b). It may therefore be convenient for certain users to have GGs in the LNOF instead of the GRF. Both frame transformation and gravity field analysis are covered in the HPF but are topics of their own and therefore outside the scope of this paper (see e.g. Bouman et al. 2007; Foerste et al. 2007; Migliaccio et al. 2004; Pail and Plank 2004).

The GOCE GGs are preprocessed both in quick-look (QL) and in final mode. The QL mode has a short latency (preprocessing within 1 day after data reception) to support mission operations, while the final mode has a longer latency (preprocessing within 2 weeks after data reception) and aims at providing the best GGs possible. The corrected and calibrated GGs from the QL mode are one of the inputs to monitor the overall system health, while the GGs from the final mode are used in the gravity field analysis and are a Level 2 end product. Besides the latency, the differences between the two modes lie in the more accurate input products and the more comprehensive preprocessing for the final mode. With respect to the former point, in the QL mode, a rapid science orbit is used as well as the operational Level $1 \mathrm{~b}$ data, whereas for the final mode a precise science orbit is used and consolidated Level 1b data (Visser et al. 2007). As far as the latter point is concerned, only tidal temporal corrections are computed in QL mode, whereas in final mode nontidal temporal corrections are also computed. In addition, in QL mode, the Level 1b GGs are calibrated using global gravity field models (baseline method), whereas in the final mode the gradiometer data is calibrated using GOCE SST and terrestrial gravity data as well. A flow-chart of the GG preprocessing is given in Fig. 3.

The simulated data used in this paper cover a period of 54 days, with a sampling of $1 \mathrm{~Hz}$. The data were generated using ESA's GOCE end-to-end (E2E) simulator and include GGs, GPS observations and gradiometer quaternions. The latter are derived from a combination of the gradiometer differential accelerations and star sensor observations (Cesare 2005). Realistic errors have been added to all simulated observations (Catastini et al. 2007). With respect to the GGs it has to be mentioned that four of them, $V_{X X}, V_{Y Y}, V_{Z Z}$ and $V_{X Z}$, have high accuracy, whereas $V_{X Y}$ and $V_{Y Z}$ are less accurate, which is a consequence of the gradiometer configuration that consists of six three-axis accelerometers. Each accelerometer has two ultrasensitive axes and one less sensitive axis, because they are built and tested on Earth in a 1- $g$ environment (ESA 1999). The E2E data were used to compute precise science orbits (Visser and van den IJssel 2000), which served as input as well. The global gravity field model underlying the E2E computations is EGM96 complete to degree and order 360 (Lemoine et al. 1998), while the global model for the model GGs is EIGEN-GL04C complete to degree and order 360 (Foerste et al. 2007). The simulated data are just one realisation of the GOCE system model based on the best effort to simulate the mission. The characteristic of the true GOCE data may of course differ from what is presented here.

\section{Corrections for temporal gravity field variations}

\subsection{Tidal temporal gravity field variations}

For the tidal GG correction, we consider a number of tidal effects acting on the gradiometer, which are all second-order derivatives of the astronomical tide-generating potential, the indirect solid Earth tide deformation potential, the self attraction and loading deformation potential and the pole tide potential. The astronomic tide-generating potential requires knowledge of planetary ephemeris data including position knowledge of the gradiometer. For this correction, we make 


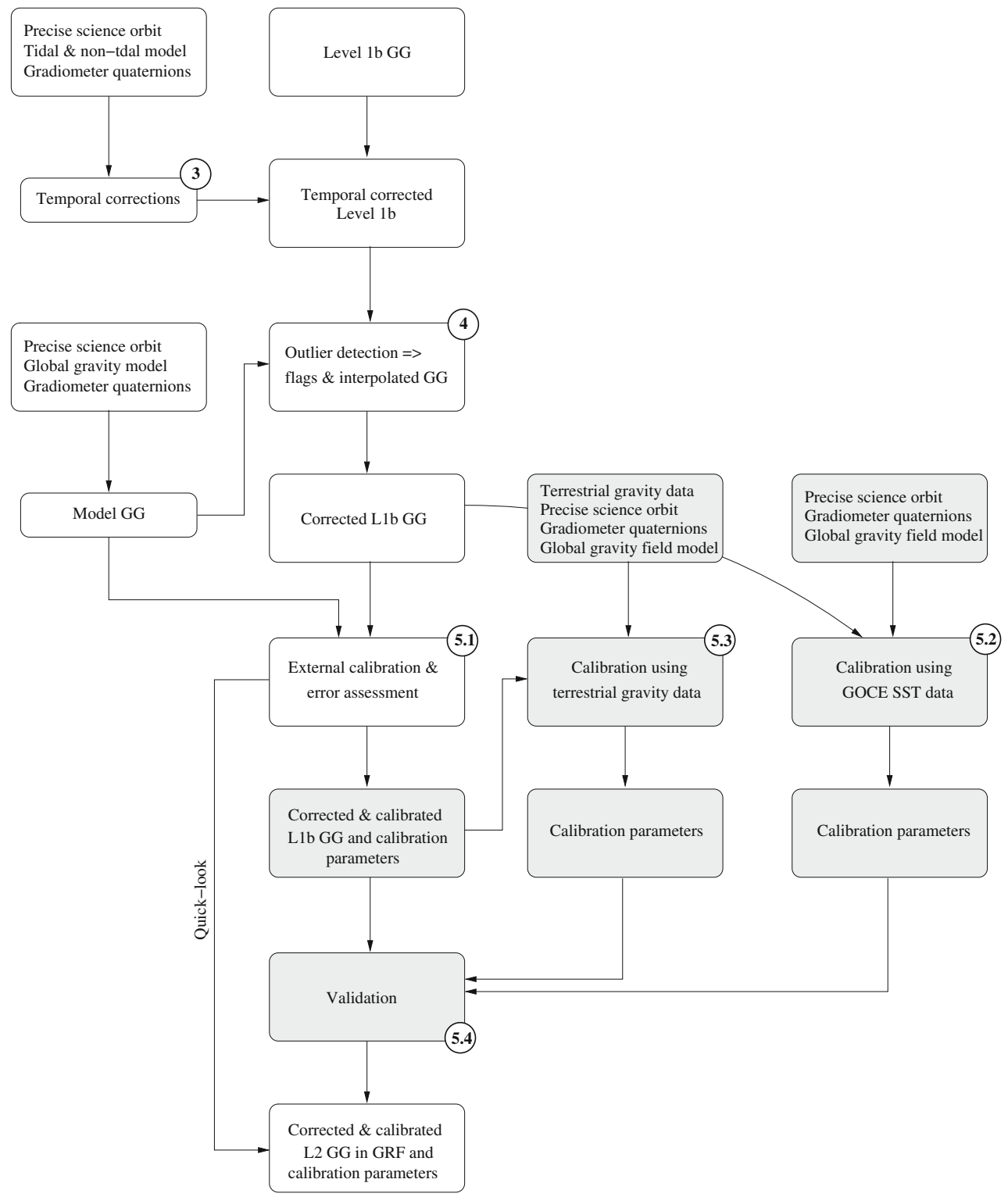

Fig. 3 Flow chart of gravity gradient preprocessing in the GOCE HPF. The blocks in grey are part of the final mode only; the numbers refer to the sections in which the algorithms are discussed

use of the JPL DE 405 planetary ephemeris dataset described by Standish (1998) and also trajectory information obtained from the GPS receiver on GOCE.

The Earth will elastically deform because of the presence of tidal forces, and the deformation effect will result in an induced potential, which can be obtained by a suitable convolution of the astronomic tide potential. In (Lambeck 1988), it is explained that the induced potential is obtained by a suitable scaling factor in the form of a $k_{n}$ Love number, where the IERS conventions are used for the definition of the required Love numbers (IERS Conventions 2004).

Tides in the oceans follow from the Laplace tidal equation where the forcing terms contain a combination of the astronomic tide generating potential and the solid Earth tide induced potential and a self attraction and loading (SAL) potential. To model the SAL potential, we use a tide model based on constants observed by satellite altimetry as described in the GOT00.2 model, which is a follow up of the procedures described by Schrama and Ray (1994). The resulting tidal water movements result in temporal variations of water masses resting (loading) on the lithosphere as described by Farrell (1972). A suitable convolution of the ocean tide grids contained in the GOT00.2 model results in a SAL potential relevant for this paper. Required for this procedure are suitable load Love numbers as mentioned by Farrell (1972).

Finally, there is a pole tide potential, which follows from the variations in Earth rotation modelled by polar motion 
parameters $x_{\mathrm{p}}$ and $y_{\mathrm{p}}$ (see also Lambeck (1988)). This results in an induced potential, which is taken into account. The IERS provides on their web portal access to C04 Earth orientation parameter tables. Input to the pole tide algorithm are differences between polar motion values and the mean definition of the pole as specified in the IERS conventions.

\subsection{Nontidal temporal gravity field variations}

GOCE has been designed to observe the static part of the Earth gravity field. To take into account temporal and spatial aliasing due to the space-time sampling of the GOCE orbit as well as due to the planned mission profile of GOCE with two 6-monthly measurement phases interrupted by a hibernation phase of 5 months, the nontidal temporal gravity field variations have to be estimated and corrections to the GGs have to be computed. For this we take into account the most prominent high-frequency signals from atmospheric and oceanic mass variations as well as seasonal variations derived from a GRACE gravity field time series. For the latter, it is assumed that it contains information about seasonal time variable gravity signal from all other sources (continental water, ice mass variations, solid Earth mass variations). In our processing chain, a set of gravity potential correction coefficients is computed for every $6 \mathrm{~h}$, which further is used to compute gravity gradient corrections for all six tensor elements.

Atmospheric and oceanic high-frequency mass variations. The mean mass distribution of oceans and atmosphere by definition belongs to the static part of the Earth gravity field. Therefore, only deviations from this mean have to be taken into account. It was decided to use the mean atmosphere and ocean computed from the years 2004-2007 to define a mean value, because this time window is close to the GOCE measurement phases (launch foreseen for early 2009). As input fields we use atmospheric data from the operational analysis of the European Centre for Medium Range Weather Forecasts (ECMWF) (see ECMWF 2007), and the baroclinic ocean model for circulation and tides (OMCT), which has been further developed from the Hamburg ocean primitive equation model (HOPE). For more details on OMCT and HOPE (see Drijfhout et al. 1996; Wolff et al. 1996; Thomas 2002; Dobslaw and Thomas 2007). The procedure applied is identical to the one used for the GRACE dealiasing (see Flechtner 2007). For the atmosphere, a full vertical integration is performed to compute the centre of mass of the atmospheric column, which is below the satellite. This is required to reach the ultimate accuracy. The sequence of formulas applied to the atmosphere is

$$
T_{\mathrm{v}}=(1+0.608 S) T ; \quad P_{k+1 / 2}=a_{k+1 / 2}+b_{k+1 / 2} P_{\mathrm{S}}
$$

$$
\begin{aligned}
& \Phi_{k+1 / 2}=\Phi_{S}+\frac{1}{\gamma} \sum_{j=k+1}^{N_{\text {level }}} R_{\mathrm{dry}} T_{\mathrm{v}} \ln \frac{P_{j+1 / 2}}{P_{j-1 / 2}} \\
& C_{n m}=l_{n} \iint_{\text {Earth }} h_{n} P_{n m}(\cos \theta) \cos m \lambda \sin \theta \mathrm{d} \theta \mathrm{d} \lambda \\
& S_{n m}=l_{n} \iint_{\text {Earth }} h_{n} P_{n m}(\cos \theta) \sin m \lambda \sin \theta \mathrm{d} \theta \mathrm{d} \lambda
\end{aligned}
$$

where

$$
l_{n}=\frac{a^{2}\left(1+k_{n}\right)}{(2 n+1) G M}
$$

$h_{n}=\int_{P_{\mathrm{S}}}^{0}\left(\frac{a}{a-\Phi}+\frac{\xi}{a}\right)^{n+4} \mathrm{~d} P-P_{\mathrm{Int}}^{\mathrm{Ref}}$

and

$S$ specific humidity at model level,

$T$ temperature at model level,

$T_{\mathrm{v}}$ virtual temperature at model level,

$a_{k+1 / 2}, b_{k+1 / 2}$ coefficients for computing pressure on model

levels (defined for atmospheric model),

$P_{\mathrm{S}}$ surface pressure,

$P_{k+1 / 2}$ pressure at model level,

$k$ model level number,

$R_{\text {dry }}$ gas constant for dry air,

$\gamma$ standard gravitational acceleration,

$\Phi_{S}$ geopotential height at Earth surface,

$\Phi_{k+1 / 2}$ geopotential height at model level,

$N_{\text {level }}$ number of model levels,

$\xi$ geoid height,

$P_{\text {Int }}^{\text {Ref }}$ vertically integrated mean for years 2004-2007,

$C_{n m}, S_{n m}$ spherical harmonic coefficients,

$n, m$ degree and order,

$a$ semi major axis of reference ellipsoid,

$k_{n}$ loading Love numbers in order to take into account the loading effects,

$G M$ gravity constant times mass of the Earth,

$P_{n m}$ associated Legendre polynomials,

$\theta, \lambda$ geographical colatitude, longitude.

The full derivation of these formulas can be found in (Flechtner 2007).

This means for the atmospheric component the following four parameters are needed from the global atmospheric model on $1 \times 1$ degree grids: (1) surface pressure, (2) geopotential height at the Earth surface, (3) temperature at vertical model levels (currently these are 91 for the ECMWF model) and (4) specific humidity at vertical model levels. In addition, a geoid is required, which is computed from a recent global gravity field model. After the vertical integration has 
been performed (inner integral, Eq. 6) the atmospheric part has to be combined with the ocean before a spherical harmonic analysis is done.

The OMCT ocean model also is driven by a set of atmospheric parameters taken from the ECMWF operational model. This means atmosphere and ocean mass variation estimates are based on the same input model, which ensures compatibility of both sources. For the ocean model, the following parameters are needed: (1) surface pressure, (2) wind speed in $u$ - and $v$-direction (North-South and East-West), (3) sea surface temperature, (4) temperatures at 2 and $10 \mathrm{~m}$ levels and (5) freshwater fluxes from precipitation minus evaporation. The model internally runs with $30 \mathrm{~min}$ step size and provides global estimates of the ocean bottom pressure for the same time steps as the atmospheric parameters are available (at 0, 6, 12 and $18 \mathrm{~h}$ UTC for each day). Because the model provides ocean bottom pressure, which is composed of the sum of pressure caused by the water column and by the atmospheric column above the point on the sea floor, the atmosphere has to be taken out again by subtracting the vertically integrated component. By this approach, it is ensured that both the impact of the centre of mass of the atmosphere and the compensation between atmosphere and ocean (in the sense of the inverse barometer effect) are taken into account. From this combined pressure field, the chosen mean (computed in the same way as the actual field) is subtracted and a spherical harmonic analysis is performed to compute potential coefficients for the gravitational attraction of the residual atmosphere and ocean pressure. For GOCE, it is sufficient to limit the spherical harmonic series to a maximum degree and order 180 , because the signal strongly decreases for higher resolution and then is far below the GOCE sensitivity (see Fig. 4). A set of coefficients is estimated for each 6-hourly time step.
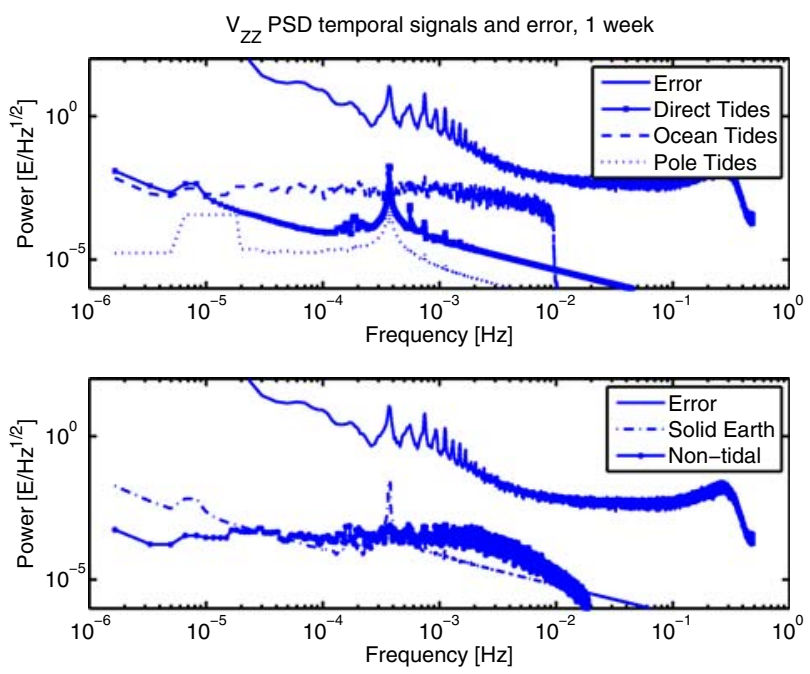

Fig. 4 PSD of one week of $V_{Z Z}$ gravity gradient error and temporal signals
Seasonal mass variations from GRACE. In context of the GRACE gravity field processing, the same strategy for highfrequency atmospheric and oceanic mass variation based on the same input data is applied as previously described for GOCE. This means a monthly time series from GRACE does not show atmospheric and oceanic mass variations, but only mass variation caused by other phenomena. As GOCE is targeting on the static field, the GRACE time series provides valuable information to take into account seasonal effects from these other sources. From many analyses, it is well known that the variations of continental water storage as well as ice mass variations are the dominant signals in the GRACE time series (see e.g. Schmidt et al. 2006; Velicogna and Wahr 2006). GOCE may also be sensitive to these signals and therefore should be taken into account for the nontidal time variable correction. The GRACE-derived correction is computed in the following way. For a multiyear monthly GRACE time series (e.g. GFZ Release 4 solutions ${ }^{1}$ ), we fit an annual cosine curve for each coefficient of the spherical harmonic series from degree 2 up to degree 20 and estimate annual amplitude and phase lag. The maximum degree was chosen to avoid the influence of less accurately determined coefficients from the GRACE models to the GOCE solution (e.g. striping features). In the final step, we interpolate for each time step of the combined atmospheric and oceanic coefficients (see above) and for each coefficient between degrees 2 and 20 the annual curve and add this as additional correction to the combined atmosphere ocean nontidal mass variations. In other words, we add an additional annual signal to each of the specified coefficients, which dominantly is caused by hydrological and ice mass variations. By this approach, it is avoided that, due to the mission profile, a final GOCE model would have a seasonal bias, because all observations are taken within the same seasons.

Nontidal gravity field product. Within the GOCE HPF, the spherical harmonic series of the nontidal gravity potential is stored every $6 \mathrm{~h}$ as internal product and further used for gravity field processing as well as for computation of gradient corrections. In addition, it is planned to make this product also available to the GOCE user community to apply it for gravity field determination with GPS data (high-low satellite-to-satellite tracking).

\subsection{Temporal gravity field variations and GG error}

In Fig. 4 the PSDs of the $V_{Z Z}$ error as well as the different temporal gravity field constituents are shown. In general, all temporal signals at satellite altitude are well below the GG error, which is consistent with those by Abrikosov et al. (2006), (see also Schrama 1995). It may therefore be argued

\footnotetext{
${ }^{1}$ http://icgem.gfz-potsdam.de/ICGEM/ICGEM.html.
} 
that temporal corrections need not be applied to the GGs. Nevertheless, there are a number of arguments in favour of the application of temporal corrections to the GGs. First, temporal gravity gradient signals have a systematic nature whereas the observation errors are anticipated to be more stochastic. Second, the dealiasing with validated models and data, as is done here, should improve the stochastic behaviour of the gradiometer data (Abrikosov et al. 2006). Finally, temporal corrections are applied to the GOCE SST data that is used both in the orbit determination and in the gravity field analysis, which combines the GOCE SST and gradiometer data. The temporal long wavelength part of the gravity field may be well visible in the GOCE SST data and to be consistent the GOCE GGs are corrected for the temporal effects. Also note that the GG temporal corrections will be given as separate entries in the final gravity gradient product. End users can therefore decide to apply the temporal corrections or not (Gruber et al. 2007a).

\section{Outlier detection}

The GGs data screening method that is adopted for the diagonal GGs $V_{X X}, V_{Y Y}$ and $V_{Z Z}$ in the HPF is based on three tests:

1. The trace of the gradient tensor is zero (Laplace condition);

2. The difference between the observed GGs and gradients from a selected global model is zero (test on GG anomalies);

3. The difference between interpolated and observed GG anomalies from (2) is zero.

If the trace test indicates a data point as an outlier and this is confirmed by one or both of the other two tests, it is flagged as such (see Bouman 2004). For the off-diagonal components $V_{X Y}, V_{X Z}$ and $V_{Y Z}$, the trace test is not applicable. Therefore, to flag an off-diagonal component as an outlier, both tests on the GG anomalies must indicate an outlier to flag the data point as such. Flagged data points are discarded in the external calibration and error assessment (Sect. 5). Should the number of flagged data points be larger than a certain threshold, e.g. $1 \%$ of daily arcs, then the automated process is put on hold and the data will be examined in more detail.

Other methods for GG outlier detection as well as a comparison of different methods are discussed in (Bouman et al. 2005; Kern et al. 2005). The outlier detection method implemented in the GOCE HPF has been updated with respect to (Bouman 2004) to account for the possibly large long wavelength errors on the GGs and to improve the robustness of the method, which is described below. In addition, simulation examples are given.

\subsection{Method}

Hypothesis test with condition equations. The outlier detection relies on data snooping in which condition equations are used (Teunissen 2000). The observation vector $\mathbf{y}$ contains the GGs (observed or modelled) whose errors are assumed to be normally distributed with known error variance matrix $\mathbf{Q}_{y}$ :

$\mathbf{y} \propto N\left(E\{\mathbf{y}\}, \mathbf{Q}_{y}\right)$

with $E$ the expectation operator. All single observations will be tested for outliers. The hypothesis

$H_{0}: \mathbf{B}^{T} E\{\mathbf{y}\}=\mathbf{0}$

will be tested against the alternative hypothesis

$H_{A}: \mathbf{B}^{T} E\{\mathbf{y}\}=\mathbf{c}_{t} \nabla, \nabla \neq 0$

where $\mathbf{B}^{T}$ is the condition equation matrix, $\mathbf{c}_{t}=\mathbf{B}^{T} \mathbf{c}_{y}$ and $\mathbf{c}_{y}$ is a unit vector with 1 at row $i$ if the $i$ th observation is to be tested, and $\nabla$ is an outlier with unknown size. In the condition equation, $\mathbf{B}^{T}$ has $b$ rows, the number of condition equations, and it has $m$ columns, the number of observations. It can be shown that $H_{0}$ will be rejected if (Teunissen 2000)

$w<-k_{\alpha}$ or $w>k_{\alpha}$

with

$w=\frac{\mathbf{c}_{t}^{T} \mathbf{Q}_{t}^{-1} \mathbf{t}}{\sqrt{\mathbf{c}_{t}^{T} \mathbf{Q}_{t}^{-1} \mathbf{c}_{t}}}$

where $\mathbf{t}=\mathbf{B}^{T} \mathbf{y}$ is the vector of misclosures, $\mathbf{Q}_{t}=\mathbf{B}^{T} \mathbf{Q}_{y} \mathbf{B}$ and $k_{\alpha}$ is the critical value that depends on the significance level $\alpha$. The random variable $w$ is the $w$ test statistic and has a standard normal distribution under $H_{0}$.

Median absolute deviation. In the outlier detection method as developed by Bouman (2004) the median of the GG trace or GG anomalies was subtracted to account for the GG biases and typical data windows of 1 day to 1 month were used. However, the $1 / f$ GG error may cause large differences between measured and model GGs also at other frequencies than at $0 \mathrm{~Hz}$ at which the bias is manifest, while also the data window may be too long. To compensate for the long wavelength errors, one could high-pass filter the GGs. A disadvantage is that in the presence of outliers, the filtering may redistribute their signal power to adjacent observations, which is undesirable. Therefore, a local median is subtracted from the gradients or trace time series using short data windows. The local median $L_{\mathrm{m}}(y)\left(t_{i}\right)$ is the median of the values

$$
\left\{y\left(t_{j}\right), j \in[i-W / 2, i+W / 2]\right\}
$$




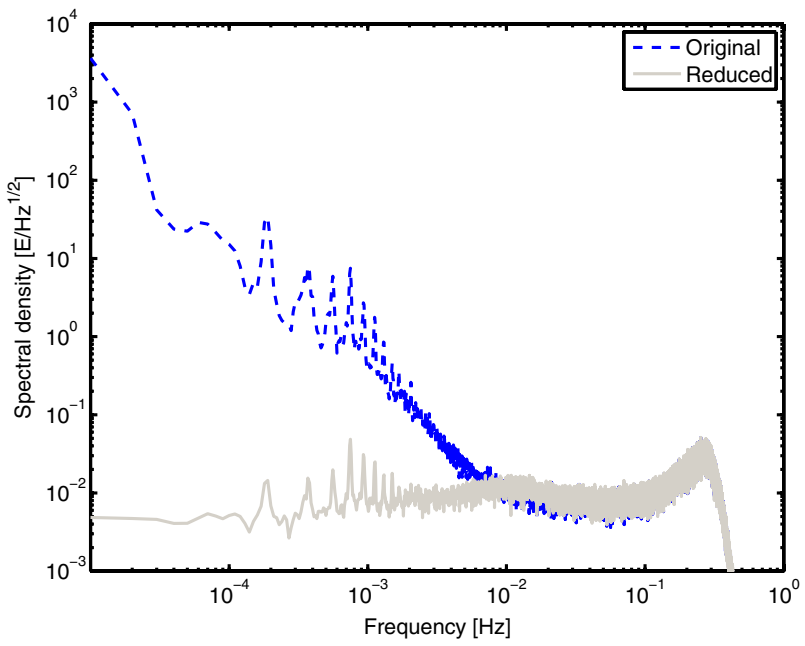

Fig. 5 PSD of the original and reduced trace. The E2E data of the first week has been used

where $W$ is a configurable window length for the local median (typical window lengths of $100 \mathrm{~s}$ are used). Here $y\left(t_{j}\right)$ is either the GG anomaly or the GG trace at time $t_{j}$. Taking the difference between the original observation $y\left(t_{i}\right)$ to be tested and the local median results in a reduced $y\left(t_{i}\right)$ :

$\operatorname{Red}(y)\left(t_{i}\right)=y\left(t_{i}\right)-L_{\mathrm{m}}(y)\left(t_{i}\right)$

which leads to a kind of high-pass-filtered observation. See for example Fig. 5, which shows the PSD of the original and reduced trace of the E2E data of the first week. Clearly, the power of the reduced trace is less than the power of the original trace below $10^{-2} \mathrm{~Hz}$.

In the $w$ test statistic, error estimates of the GG anomalies or the trace are needed (see Eq. 11). The standard deviation (SD) of the trace and difference data are an error estimate of these data. However, the SD can be significantly affected by a few outliers that are very different from the rest of the data. It is therefore important to use a robust alternative. One such alternative to the $\mathrm{SD} \sigma$ is the median absolute deviation (MAD) which has the advantage that it is not sensitive to a small portion of large outliers or to heavy tails in the data. The MAD is calculated as the median of the absolute difference between a value and its local median, in other words the median of the absolute of the reduced value:

$\operatorname{MAD}(\mathbf{y})=\operatorname{median}(|\operatorname{Red}(\mathbf{y})|)$.

To calculate an estimate of the SD $\hat{\sigma}$, the MAD is multiplied by the ratio of SD and MAD for a normal distribution:

$\hat{\sigma}=r \times \mathrm{MAD}$

with $r=1.4826$ (see Hoaglin et al. 1983).
Three tests. The trace of the gravity gradient tensor is the sum of its diagonal elements

$\mathbf{y}_{\Sigma}=\mathbf{V}_{X X}+\mathbf{V}_{Y Y}+\mathbf{V}_{Z Z}$

According to the Laplace condition, the trace of the GG tensor must be zero. This equation is tested by taking the reduced trace $\operatorname{Red}\left(\mathbf{y}_{\Sigma}\right)$, and comparing it against the threshold $k \hat{\sigma}$, where $\hat{\sigma}$ is the SD estimate from MAD of the reduced trace, and $k$ is a configurable parameter setting the strictness of the criterion. If $\left|\operatorname{Red}\left(\mathbf{y}_{\Sigma}\right)\right|>k \hat{\sigma}$ for a certain epoch, the trace flag for the three diagonal elements of the GG tensor of this epoch is set.

A second test is on the GG anomalies, which are computed as the difference between the measured GGs $\mathbf{V}_{\xi \eta}$ and the modelled gradients $\mathbf{U}_{\xi \eta}$ :

$\mathbf{y}_{\xi \eta}=\mathbf{V}_{\xi \eta}-\mathbf{U}_{\xi \eta}, \xi \eta=X X, Y Y, Z Z, X Y, X Z, Y Z$

Also, for the anomaly $\mathbf{y}_{\xi \eta}$, the reduced anomaly $\operatorname{Red}\left(\mathbf{y}_{\xi \eta}\right)$ is compared against $k \hat{\sigma}_{\xi \eta}$, where $\hat{\sigma}_{\xi \eta}$ is the SD estimate of $\mathbf{y}_{\xi \eta}$, which is calculated separately for each component of the tensor. If $\left|\operatorname{Red}\left(\mathbf{y}_{\xi \eta}\right)\right|>k \hat{\sigma}_{\xi \eta}$ for a certain epoch, the difference flag for this component is set.

The third test overall and the second test on the GG anomalies is to compare $\mathbf{y}_{\xi \eta}$ with an interpolated value using Overhauser splines (Overhauser 1968), which is based on the two epochs before and the two epochs after the epoch under test. If the difference between $\mathbf{y}_{\xi \eta}$ and the interpolated $\mathbf{y}_{\xi \eta}^{\text {int }}$ is larger than $k \hat{\sigma}_{\xi \eta}$, the interpolation flag is set (for details see Bouman 2004).

The advantage of the trace test is that no model errors are involved. This in contrast to the test on GG anomalies. The differences between the observed GOCE GGs and the model GGs may be systematic, because the underlying global gravity field model may contain errors that are correlated with, for example, signal over the Himalayas due to data deficiencies (see also Sect. 4.2). These systematic errors may cancel in the spline interpolation which is therefore an advantage of this method. A disadvantage of the spline interpolation is that different epochs are combined, which may lead to ambiguities between epochs, while offsets may cancel because of the interpolation. On the other hand, in the trace test and in the test using GG anomalies, outliers can be searched for at each individual time step. A disadvantage of the trace test is that the off diagonal GGs cannot be tested, while the method may not unambiguously distinguish between outliers on the diagonal GGs. However, with the test on GG anomalies and the spline interpolation, it is possible to test all six GGs and no ambiguities between GGs occur. All in all, the three methods are complementary. 
Table 1 A posteriori SD $\hat{\sigma}(\mathrm{mE})$ of the reduced quantities

\begin{tabular}{lllllll}
\hline $\mathbf{y}_{\Sigma}$ & $\mathbf{y}_{X X}$ & $\mathbf{y}_{Y Y}$ & $\mathbf{y}_{Z Z}$ & $\mathbf{y}_{X Y}$ & $\mathbf{y}_{X Z}$ & $\mathbf{y}_{Y Z}$ \\
\hline 10.7 & 9.2 & 7.0 & 9.8 & 200 & 7.4 & 285 \\
\hline
\end{tabular}

Table 2 Flags set for outlier-free data (\%)

\begin{tabular}{lllll}
\hline GG & Trace & Difference & Interpolation & Combined \\
\hline Trace & 0.36 & - & - & - \\
$V_{X X}$ & 0.30 & 11.22 & 0.00 & 0.02 \\
$V_{Y Y}$ & 0.30 & 3.65 & 0.00 & 0.02 \\
$V_{Z Z}$ & 0.30 & 12.31 & 0.00 & 0.02 \\
$V_{X Y}$ & - & 0.32 & 0.00 & 0.00 \\
$V_{X Z}$ & - & 14.65 & 0.00 & 0.00 \\
$V_{Y Z}$ & - & 0.33 & 0.00 & 0.00 \\
\hline
\end{tabular}

\subsection{Numerical examples}

In this section, the characteristics of the outlier detection method will be illustrated numerically. First an example is given of GG data without outliers and secondly GG data with outliers are discussed. The first 28 days of the full 54-day data set were used, consisting of 2,419,200 epochs. The critical value $k$ has been set to $k=3$, which corresponds to a significance level of $0.27 \%$. The estimated a posteriori SDs from Eq. 15 are given in Table 1. The SD of the reduced, accurate GGs is approximately $10 \mathrm{mE}$, while the $\mathrm{SD}$ of the less accurate GGs is a factor of 20-30 times larger.

In Table 2, the percentage of detected outliers is shown for the data without outliers. Columns two to four show the outlier percentage for the three individual criteria, while the last column shows the combination, that is, how many outliers are flagged in the end. To start with the latter, we see that the combination of the three tests yields very small percentages of erroneously flagged outliers. For all GGs it is $0.02 \%$ or less, which indicates that at most every 2 out of 10,000 observations are erroneously flagged as an outlier. The percentage of erroneous flags is low and seems to be acceptable. It could be reduced even further by choosing a larger critical value $k$. However, the smallest outlier that can be detected has size $\sim k \hat{\sigma}$ and a larger critical value would therefore mean that also the smallest outlier that can be detected becomes larger, which may be a drawback.

If we now turn to the three individual tests, then we see that the percentage of flagged data points in the trace test, $0.30 \%$ for the diagonal GGs, is close to the significance level of $0.27 \%$, which is to be expected if the errors are normally distributed. The flag percentages of the trace and the individual diagonal GGs differ because an iterative process is used. First, all $V_{X X}, V_{Y Y}$ and $V_{Z Z}$ GGs for a certain epoch are flagged if the reduced trace is larger than the threshold $\left|\operatorname{Red}\left(y_{\Sigma}\right)\left(t_{i}\right)\right|>k \hat{\sigma}$. Second, all flagged data are checked

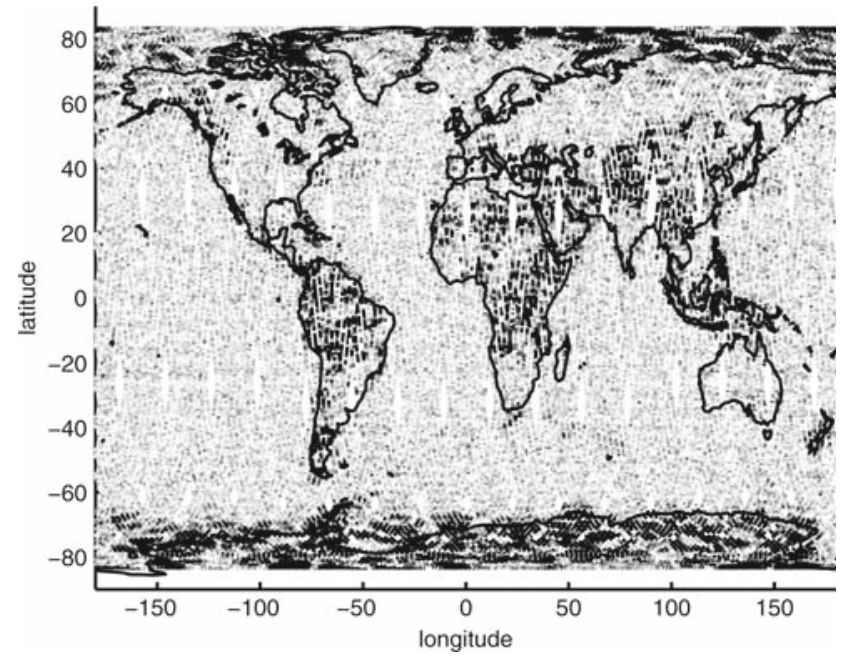

Fig. 6 Geographical plot of $\left|\operatorname{Red}\left(\mathbf{y}_{Z Z}\right)\right|$

again replacing all but one flagged data points with interpolated values. This may lead to a confirmation of the flag of the data point under test, but the flag may also be removed.

The outlier percentage of the difference test is large for the accurate GGs $V_{X X}, V_{Y Y}, V_{Z Z}$ and $V_{X Z}$, while it is close to the significance level for the less accurate GGs $V_{X Y}$ and $V_{Y Z}$. The differences between the simulated GOCE GGs and the model GGs are mainly determined by the GOCE GG noise, which is quite large for the latter two gradients (cf. Table 1). On the other hand, for the accurate GGs the differences between the GOCE and model GGs are also due to the differences between EGM96 and EIGEN-GL04C, which are not normally distributed but show strong geographical correlation. In the simulation, EGM96 is the model underlying the GOCE GGs, while EIGEN-GL04C has been used to compute the model GGs. Figure 6 displays the $\left|\operatorname{Red}\left(\mathbf{y}_{Z Z}\right)\right|$, which is large for high latitudes, South America, Africa and parts of Asia. One of the main improvements of EIGEN-GL04C over EGM96 is the inclusion of GRACE data in the former model (Foerste et al. 2007). The differences between the two models are especially visible where the quality and data distribution of EGM96 are weak, that is, the polar caps, previously not very accurately resolved by satellite-only models due to the relatively small inclinations of the analyzed satellites, and the continents of Africa, Asia and South America, which are only in-homogeneously covered by satellite tracking stations and surface gravimetric data (Foerste et al. 2007). Because of the relatively poor data quality in the mentioned regions for EGM96, the additional GRACE data may explain the larger differences in these regions. The smaller percentage of erroneous flagged outliers for $V_{Y Y}$ in the difference test may be explained by the weaker cross-track sensitivity of GRACE (approximately the same as the GOCE $Y$-direction): the improvements due to GRACE are especially visible in the $X$ and $Z$-direction. 
Table 3 Flags set for data with outliers (\%)

\begin{tabular}{lllll}
\hline GG & Trace & Difference & Interpolation & Combined \\
\hline Trace & 0.54 & - & - & - \\
$V_{X X}$ & 0.35 & 11.27 & 0.06 & 0.08 \\
$V_{Y Y}$ & 0.35 & 3.71 & 0.07 & 0.08 \\
$V_{Z Z}$ & 0.35 & 12.34 & 0.06 & 0.08 \\
$V_{X Y}$ & - & 0.36 & 0.04 & 0.04 \\
$V_{X Z}$ & - & 14.68 & 0.08 & 0.08 \\
$V_{Y Z}$ & - & 0.36 & 0.03 & 0.03 \\
\hline
\end{tabular}

The number of detected outliers is (almost) identical to zero for the interpolation criterion for all GGs when no artificial outliers have been added. Because of the interpolation, correlated errors cancel. Since the GOCE gravity gradient errors are correlated along track and since the EGM96-EIGEN04C differences are correlated over the short time spans we are dealing with here (seconds), these errors cancel indeed and almost no data are flagged.

The same 28-day data set was used in the second test, except that outliers are artificially created in the data. The outliers are randomly applied, with each data point having a chance of $0.1 \%$ to be an outlier, and with a magnitude that is uniformly distributed on a logarithmic scale between $0.01 \mathrm{mE}$ and $100 \mathrm{E}$. As the outlier percentage is $0.1 \%$, the maximum increase in flags set with respect to the outlier free case is also $0.1 \%$ for all individual GGs, whereas the maximum increase is $0.3 \%$ for the trace test because it uses the sum of the three diagonal GGs. The percentage of flagged outliers is shown in Table 3. These flags are either erroneous or they do flag an outlier. In the current example, the percentage of erroneous flags is almost equal to the percentages presented in Table 2 and are therefore not shown. This implies that the percentage of correctly set outlier flags can be derived by subtracting the numbers of Table 2 from those of Table 3 . For the combined case, this means that the percentage of correct flags varies from roughly 0.03 to $0.08 \%$, which in turn means that 30 $80 \%$ of the outliers have been detected, as the total outlier percentage is $0.1 \%$.

However, the percentage of detected outliers may not be the most important number to consider, because this strongly depends on the size of the generated outliers and these can be as small as $0.01 \mathrm{mE}$ in our example. Instead, the minimal detectable outlier may be an illustrative number, and it can be derived by multiplying the a posteriori SDs of Table 1 with the critical value $k=3$. Figures 7 and 8 display the detected as well as the undetected outliers for $V_{Z Z}$ and $V_{X Y}$, respectively. Naturally, the threshold for the accurate GG $V_{Z Z}$ is much smaller than that for the less accurate GG $V_{X Y}$, which in turn has an effect on the number of detected outliers (see also Table 3, last column). Again, we remark that the smallest outlier that can be detected has size $\sim k \hat{\sigma}$. Should we wish

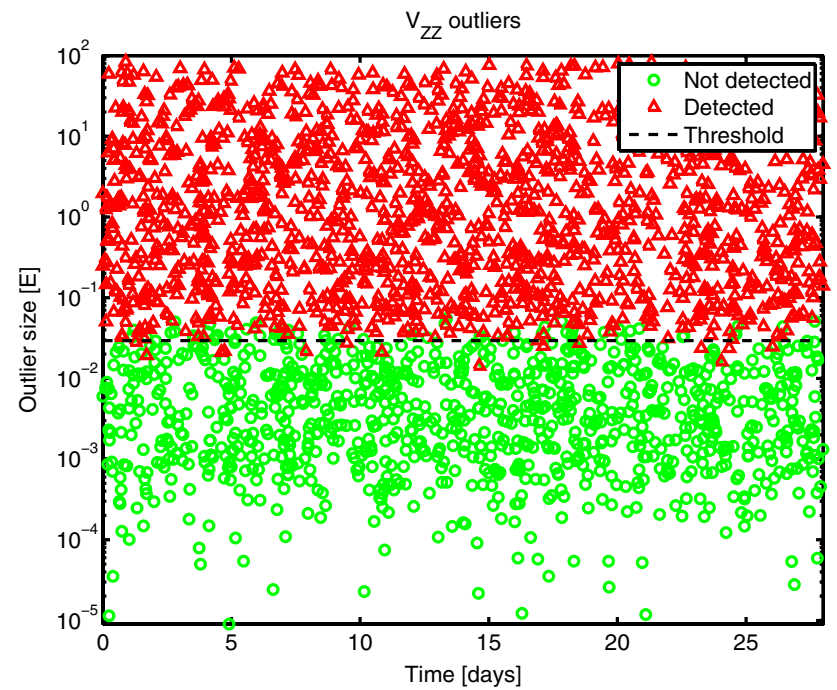

Fig. 7 Detected and undetected $V_{Z Z}$ outliers

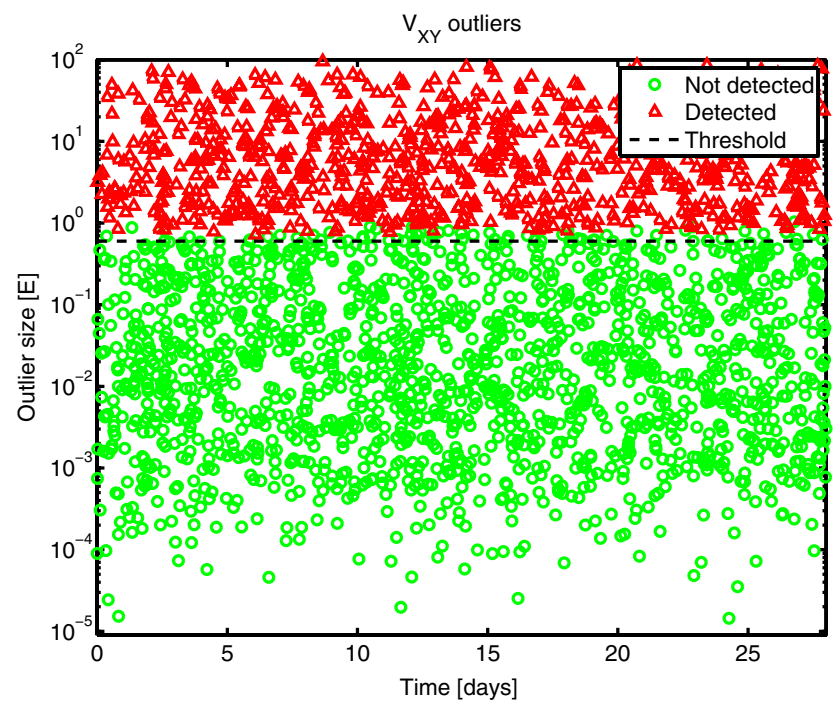

Fig. 8 Detected and undetected $V_{X Y}$ outliers

to determine smaller outliers then the critical value has to be choosen smaller (since we have little influence on $\hat{\sigma}$ ), with consequently a larger percentage of erroneous outliers that will be detected.

\section{External calibration}

Three different methods are used in the external calibration of the GOCE GGs. The baseline method is the calibration using a global gravity field model, which is used to compute model GGs at satellite level and these GGs serve as reference values for the GOCE GGs to determine, for example, GG scale factors. A second calibration method uses GOCE SST data and GOCE GGs to estimate the spherical harmonics of a 
global gravity field model, truncated at degree and order 80 , together with calibration parameters. The third calibration method uses terrestrial gravity data. Least-squares collocation (LSC) is used to compute GGs at satellite altitude from the gravity data in selected regions. These GGs serve as reference values with which the GOCE GGs may be calibrated. In Sect. 5.1-5.3, the three calibration methods are discussed and the differences obtained with Bouman et al. (2004) are highlighted. Section 5.4 discusses the cross validation between the external calibration methods.

\subsection{Global gravity field models}

The calibration using global gravity field models relies on the comparison of GOCE GGs with model GGs. The calibration model we use is (see also Bouman et al. 2004),

$$
\begin{aligned}
E\{\mathbf{y}(t)\}= & \lambda \mathbf{y}_{s}(t)+\Delta \mathbf{y}+\mathbf{y}^{\prime} \cdot t \\
& +\sum_{k=1}^{K}\left[a_{k} \cos k \omega(t)+b_{k} \sin k \omega(t)\right]
\end{aligned}
$$

with $E$ the expectation operator, $y$ GOCE GGs-corrected for temporal gravity and with outliers flagged, $\lambda$ scale factor, $\mathbf{y}_{\mathrm{s}}$ the "true" GGs (s for static), $\Delta \mathbf{y}$ bias, $\mathbf{y}^{\prime}$ trend, $\omega=2 \pi t / T$, $t$ time, $T$ mean orbital period and $a_{k}, b_{k}$ Fourier coefficients. The number of unknowns is $3+2 K$, that is, a GG scale factor, bias, trend and $2 K$ Fourier coefficients. In addition, also the "true" GGs $\mathbf{y}_{s}$ are unknown. Approximations $\mathbf{y}_{m}$ to these GGs can be computed, using a global gravity field model, which are either taken as deterministic because they can be expected to be accurate at long wavelengths below the MB where most of the signal power is (Bouman et al. 2004) or as stochastic taking the model error into account (Bouman and Koop 2003a). In the latter approach, the linearised system of equations may become very large due to the error correlations. Nevertheless, we have followed this approach, neglecting all error correlations, as it may formally be a more correct procedure. The differences between the two approaches have been reported to be small (Bouman et al. 2004). The nonlinear model is

$$
\begin{aligned}
E\left\{\mathbf{y}(t)-\lambda \mathbf{y}_{m}(t)\right\}= & \Delta \mathbf{y}+\mathbf{y}^{\prime} \cdot t+\sum_{k=1}^{K}\left[a_{k} \cos k \omega(t)\right. \\
& \left.+b_{k} \sin k \omega(t)\right] .
\end{aligned}
$$

In (Bouman and Koop 2003a; Bouman et al. 2004) GG biases, scale factors and empirical Fourier coefficients at one to four CPR are estimated using unfiltered GOCE and model GGs $\mathbf{y}$ and $\mathbf{y}_{m}$. However, if the $1 / f$ error dominates the signal for low frequencies, which is the case for the current simulated data set, such a direct comparison does not yield reliable calibration parameters (see also Fig. 2). It is therefore necessary to high-pass filter both the GOCE GGs and the model

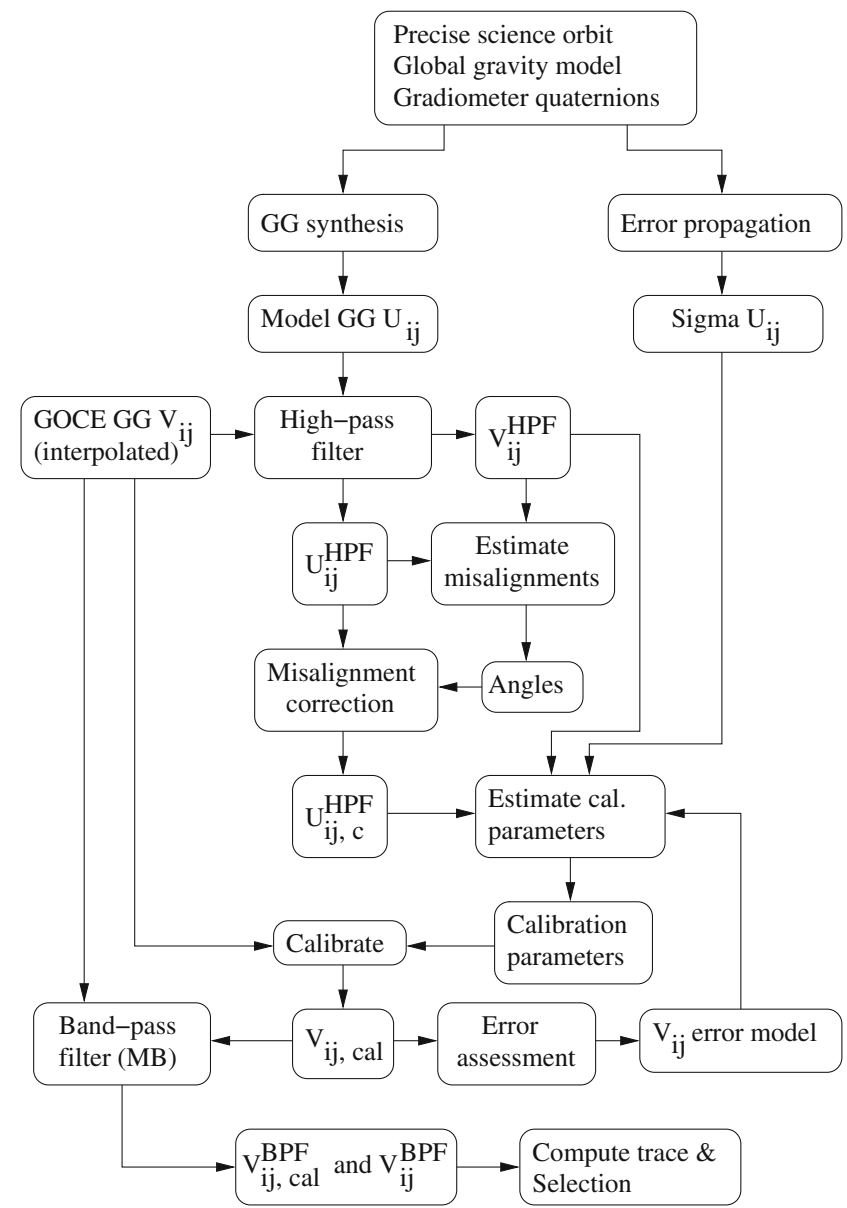

Fig. 9 Flow chart of calibration using global gravity field models

GGs before the actual calibration. In addition, to compute the model GGs in the GRF attitude information is required, that is, GOCE quaternions are needed. These quaternions combine star sensor and gradiometer data, and therefore, the model GGs may be affected by instrument misalignments (see Pail 2005) for which we may wish to correct.

A flow chart of the calibration using global gravity field models is given in Fig. 9. Model GGs as well as their predicted uncertainties are computed using a global gravity field model, gradiometer quaternions and a precise science orbit. The model GGs and the GOCE GGs are high-pass-filtered and these high-pass-filtered gradients are used to computed corrections for misalignments. The GOCE GGs may have been flagged for outliers or may contain data gaps. Interpolated GGs are computed for these epochs to allow filtering. The interpolated values are flagged and are discarded in subsequent computations except in the filtering. The corrected and high-pass-filtered model GGs are used to determine calibration parameters of the high-pass filtered GOCE GGs. The calibration parameters are applied to the unfiltered GOCE GGs and their error is assessed. In addition, the trace in the MB of the GOCE GGs and the calibrated GGs is computed 
to ensure that in the end the GG set with the smallest error in the MB is selected. Below, the high-pass filter is described in more detail, as are the method to correct for the misalignments, the error assessment and the selection method. In addition, numerical examples are given which highlight the estimation of the misalignments and the interpolation of data gaps.

High-pass filter and selection. A second order high-pass Butterworth filter is applied to the GG time series in forward and reverse direction to remove any phase distortion, which in effect doubles the filter order. The filter cut-off frequency should be chosen such that the $1 / f$ error is suppressed to a sufficiently low level, while leaving enough signal power at one and two CPR. The optimal cut-off frequency is searched in the range between $10^{-4}$ and $10^{-3} \mathrm{~Hz}$ with a step size of $10^{-4} \mathrm{~Hz}$. The lower bound of $10^{-4} \mathrm{~Hz}$ roughly corresponds to one $\operatorname{CPR}\left(1 / 5,400 \mathrm{~s} \approx 1.9 \times 10^{-4} \mathrm{~Hz}\right)$, while the upper bound of $10^{-3} \mathrm{~Hz}$ is well below the lower bound of the MB $\left(5 \times 10^{-3} \mathrm{~Hz}\right)$. For each cut-off frequency, calibration parameters of the GOCE GGs are determined using the model GGs, which have been corrected for instrument misalignments (see below).

The calibration parameters, which have been determined using high-pass-filtered data, are applied to the unfiltered GOCE GGs. Next, the calibrated GGs are band-pass-filtered such that the MB is kept and the trace is computed. The cut-off frequency that gives the minimal trace SD in the MB for the calibrated GGs is considered to be optimal. This optimal trace value is then compared with the trace SD of the band-passfiltered, not externally calibrated, GOCE GGs. If the latter is larger than the former, the external data can improve upon the in-flight calibration. Our simulations show that the external calibration with global gravity field models yields GG scale factors accurate to $10^{-3}$ (see Instrument misalignments), but that in the current example the external data cannot improve upon the a priori GOCE GG scale factors.

The high-pass filter suppresses signal and error at low frequencies. Consequently, errors manifested at these frequencies, such as a GG bias and trend, cannot be estimated (well). Furthermore, if unfiltered data is used most signal power will be present at $0,1,2, \ldots, \mathrm{CPR}$ and a GG scale factor will be most visible at these frequencies. The signal at zero CPR allowed Bouman et al. (2004) to estimate a GG scale factor as well as empirical Fourier coefficients at one to four CPR. However, after applying a high-pass filter, the signal at zero CPR will be zero and most of the remaining power of the high-pass-filtered GGs will be at one to four CPR. As a result, large correlations between the GG scale factors and the Fourier coefficients may occur in the sense that they cannot be estimated seperately. Initial computations showed that this is true for the current data: the estimation of GG scale factors and Fourier coefficients leads to unreliable scale factor estimates, that is, they may differ significantly from one. The calibration is therefore limited to GG scale factors, whereas bias, trend and Fourier coeffients are not estimated.

Instrument misalignments. The model GGs used in the calibration are ideally given in the GRF. However, the gradiometer quaternions that represent the realisation of the GRF are affected by systematic and stochastic errors. Consequently, the model GGs are given in the approximated GRF. The measured GOCE GGs, in contrast, suffer from many errors, but are given in principle in the (unknown) GRF. With respect to the systematic errors, the attitude reconstruction error, expressed in terms of Euler angles, might be affected by an alignment bias, resulting mainly from the star sensor misalignments and from a potential misalignment of the star sensors and the gradiometer mounting on the platform (Cesare 2005).

Expressed in Euler angles $\epsilon=\{\alpha, \beta, \gamma\}$ the misalignment of the realized GRF with respect to the true GRF can be formulated as rotation matrix $\mathbf{R}$

$\mathbf{R}=\mathbf{R}_{1}(-\alpha) \mathbf{R}_{2}(-\beta) \mathbf{R}_{3}(-\gamma)$

leading to a misaligned GG model tensor $\mathbf{V}^{\prime}$

$\mathbf{V}^{\prime}=\mathbf{R} \mathbf{V} \mathbf{R}^{T}$

where $\mathbf{V}$ are the model GGs in the true GRF (see also Pail 2005). The misalignment angles are expected to be small, in the order of $10^{-4} \mathrm{rad}$ in the simulated data, and the approximations $\sin \epsilon=\epsilon$ and $\cos \epsilon=1$ are justified. The mean of the high-pass-filtered GGs will be zero, while the signal RMS varies from several to tens of Eötvös units. If we take for example a cut-off frequency of $10^{-4} \mathrm{~Hz}$ and apply the Butterworth filter as discussed, then the signal RMS is approximately 5, 7, 12, 3, 41 and $76 \mathrm{E}$ for $V_{X X}, V_{Y Y}, V_{Z Z}, V_{X Y}, V_{X Z}$ and $V_{Y Z}$, respectively. We therefore may neglect in addition all quadratic terms, and the following linear relation is obtained between the tensor components of the true and misaligned system

$$
\left(\begin{array}{c}
V_{X X}^{\prime} \\
V_{X Y}^{\prime} \\
V_{Y Y}^{\prime} \\
V_{X Z}^{\prime} \\
V_{Y Z}^{\prime} \\
V_{Z Z}^{\prime}
\end{array}\right)=\left(\begin{array}{cccccc}
1 & 2 \gamma & 0 & -2 \beta & 0 & 0 \\
-\gamma & 1 & \gamma & \alpha & -\beta & 0 \\
0 & -2 \gamma & 1 & 0 & 2 \alpha & 0 \\
\beta & -\alpha & 0 & 1 & \gamma & -\beta \\
0 & \beta & -\alpha & -\gamma & 1 & \alpha \\
0 & 0 & 0 & 2 \beta & -2 \alpha & 1
\end{array}\right)\left(\begin{array}{c}
V_{X X} \\
V_{X Y} \\
V_{Y Y} \\
V_{X Z} \\
V_{Y Z} \\
V_{Z Z}
\end{array}\right)
$$


Table 4 Mean and SD of GG scale factors if misalignments are estimated or not

\begin{tabular}{llllll}
\hline GG & \multicolumn{2}{l}{ Misalignments estimated } & & \multicolumn{2}{l}{ No misalignments estimated } \\
\cline { 2 - 3 } \cline { 5 - 6 } & Mean & $\sigma$ & & Mean & $\sigma$ \\
\hline$V_{X X}$ & 1.000 & $3 \times 10^{-4}$ & & 0.996 & $4 \times 10^{-4}$ \\
$V_{Y Y}$ & 0.997 & $2 \times 10^{-4}$ & & 0.994 & $3 \times 10^{-4}$ \\
$V_{Z Z}$ & 0.998 & $1 \times 10^{-4}$ & & 0.995 & $3 \times 10^{-4}$ \\
$V_{X Y}$ & 0.999 & $11 \times 10^{-4}$ & & 0.991 & $9 \times 10^{-4}$ \\
$V_{X Z}$ & 0.998 & $1 \times 10^{-4}$ & & 0.998 & $1 \times 10^{-4}$ \\
$V_{Y Z}$ & 0.998 & $1 \times 10^{-4}$ & & 0.998 & $1 \times 10^{-4}$ \\
\hline
\end{tabular}

which can be re-written as

$$
\begin{gathered}
\left(\begin{array}{c}
V_{X X}^{\prime} \\
V_{X Y}^{\prime} \\
V_{Y Y}^{\prime} \\
V_{X Z}^{\prime} \\
V_{Y Z}^{\prime} \\
V_{Z Z}^{\prime}
\end{array}\right)-\left(\begin{array}{c}
V_{X X} \\
V_{X Y} \\
V_{Y Y} \\
V_{X Z} \\
V_{Y Z} \\
V_{Z Z}
\end{array}\right) \\
=\left(\begin{array}{ccc}
0 & -2 V_{X Z} & 2 V_{X Y} \\
V_{X Z} & -V_{Y Z} & V_{Y Y}-V_{X X} \\
2 V_{Y Z} & 0 & -2 V_{X Y} \\
-V_{X Y} & V_{X X}-V_{Z Z} & V_{Y Z} \\
V_{Z Z}-V_{Y Y} & V_{X Y} & -V_{X Z} \\
-2 V_{Y Z} & 2 V_{X Z} & 0
\end{array}\right)\left(\begin{array}{l}
\alpha \\
\beta \\
\gamma
\end{array}\right)
\end{gathered}
$$

which is a system of observation equations. The unknowns are the misalignments, the observations are the model GGs $\mathbf{V}^{\prime}$ and as an approximation for $\mathbf{V}$ the GOCE GGs in the GRF are used. The misalignments thus estimated can be used in the inverse relation of Eq. 22 to correct the model GGs.

In Table 4 the scale factors for the six GGs are shown for the two cases estimating misalignments or not. For each of the 8 weeks of the E2E data set, GG scale factors were estimated. Table 4 shows the mean optimal scale factor for this period as well as the SD with respect to the mean. In general, the coestimation of misalignments improves the GG scale factors, that is, they are closer to one, while also the variation tends to be less from week to week for the diagonal GGs.

Interpolation of data gaps. In case the GG time series contains data gaps, values for the missing epochs need to be added such that an equidistant time series is obtained, which is required because filtering is applied. Linear interpolation is applied to fill in a data gap and the interpolated values are flagged and do not contribute to the calibration.
A test was performed on a subset of the E2E data (Sect. 2), that is, 1 week. Two data sets were considered for this period, one data set with the regular E2E data and one data set with data gaps introduced. For each of the 7 days a data gap was introduced, which varied in length from 7 to $25 \%$ of 1 day, whereas the lumped data gap length is $15 \%$ of 1 week. The GG scale factors for the nominal case and the data gap case do not significantly differ, that is, the differences between the GG scale factors for the two cases is below $10^{-3}$ for all six GGs. A linear interpolation of data gaps seems therefore to be sufficient.

Error assessment. In general, observational errors may be assessed by comparing the observations with predicted observations. The cross validation method presented here is updated from (Bouman and Koop 2003b), while other methods are discussed in (Albertella et al. 2000; Koop et al. 2002; Jarecki et al. 2006; Jarecki and Müller 2007).

GOCE will deliver time series of GGs along its orbit. A subset of the time series may be used to interpolate alongtrack at time $t=i$. The difference between the interpolated $V_{\xi \eta}^{\text {int }}(t=i)$ and the measured $V_{\xi \eta}(t=i)$ is due to, among others, the interpolation error and the measurement errors. If the along-track interpolation error is small enough, then the above differences could be used for error assessment. Bouman and Koop (2003b) show that the interpolation error is below the GG error in the MB using Overhauser splines if the sampling distance along track is below $5 \mathrm{~s}$ and the GGs are generated with a global gravity field model complete up to degree and order 360.

In (Bouman and Koop 2003b), a posteriori least squares errors are estimated using the model of condition equations. The condition in this case is that the differences between the measured and interpolated GGs are zero. In addition, an unbiased estimator of the variance of unit weight is computed by comparing the a priori GG error model (which includes the GG along-track error correlation) with the a posteriori errors (see also Teunissen 2000). If the estimated variance of unit weight differs too much from one, then the a priori error model will be rejected. The a priori error model will be scaled with the a posteriori variance of unit weight, and the estimation of calibration parameters is repeated with the updated error model. Bouman and Koop (2003b) used a one-sided test to detect too optimistic a priori error models, whereas in the current implementation in the HPF a two-sided test is used to detect both too optimistic and too pessimistic a priori error models.

Along-track interpolation for error assessment uses local data, which means that this method may be suited to assess the error especially for high frequencies, that is, near or inside the MB. A disadvantage may be that systematic, long wavelength errors may cancel using local interpolation methods. On the 


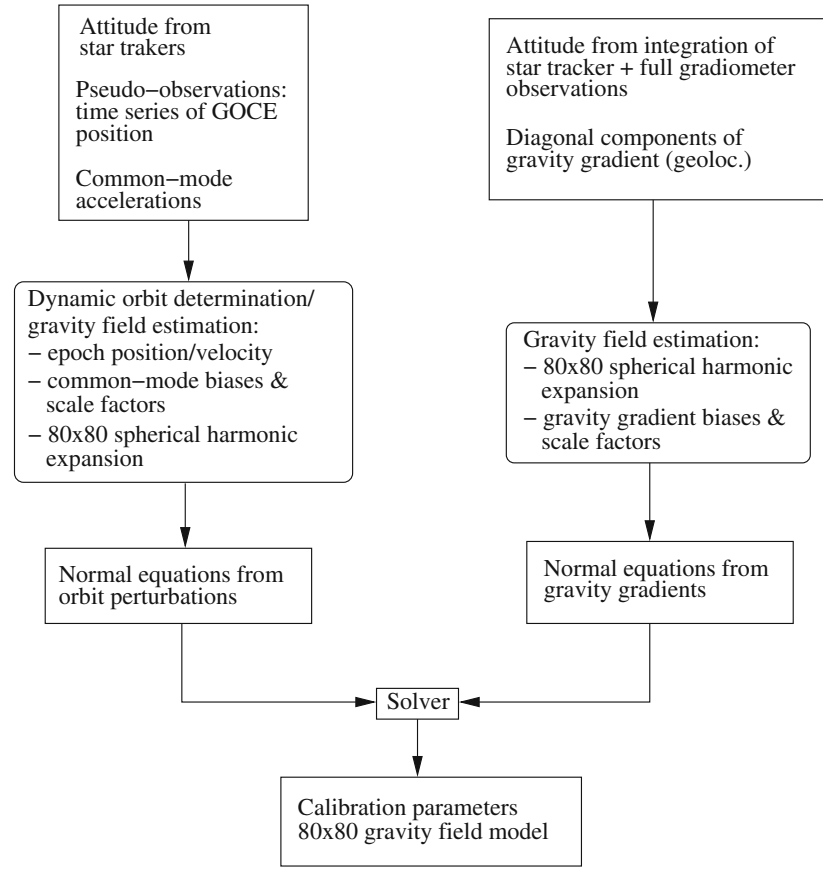

Fig. 10 Processing scheme for the GOCE gradiometer calibration from GPS (Visser 2007)

other hand, this implies that the $1 / f$ error poses no problem to the error assessment.

\subsection{GOCE SST data}

The calibration of the GGs by GPS entails the estimation of common-mode acceleration and GG calibration parameters (diagonal components only), orbital parameters and gravity field coefficients (Fig. 10, see also Visser 2007). The observables consist of time series of GG observations and orbital positions. These orbital positions are in the form of time series of highly accurate Cartesian $X, Y$ and $Z$ coordinates in an Earth-centered, pseudo-inertial reference frame. These positions are determined by reduced-dynamic orbit determination (RDOD, e.g. Yunck et al. 1994) from the GPS satelliteto-satellite tracking (SST) observations. Normal equations for a combined least-squares estimation of dynamic orbit parameters, common-mode accelerometer calibration parameters and gravity field coefficients from these orbital positions will be computed based on the numerical integration of the variational equations (Visser and van den IJssel 2000; Visser et al. 2001) with the NASA/GSFC GEODYN software package (Rowlands et al. 1995).

In addition, normal equations will be computed for gravity field coefficients and GG calibration parameters from diagonal GG observations, where these GG observations are geo-located by making use of the RDOD solution. The attitude of the satellite is derived from observations collected by the on-board star trackers, nominally enhanced by angular accelerations observed by the gradiometer. A high-pass filter is applied to the GG observations to eliminate the effect of the $1 / f$ low frequency noise. Use is made of an inversed sinc filter with a cut-off frequency of $0.1 \mathrm{mHz}$.

The method has been implemented within the HPF. The baseline is to do a calibration every 10 days, allowing the estimation of gravity field spherical harmonic coefficients to degree and order 80 . The method has been tested in detail using all anticipated instrument error sources as modelled by an end-to-end simulator (Catastini et al. 2007; Visser 2007). In addition, gravity field model errors were taken into account as well. In fact, the calibrated coefficient errors of the GGM02C model (Tapley et al. 2005) were fed to a random generator and added to the simulated true world gravity field model, which is EGM96 (Lemoine et al. 1998) in the end-to-end simulator.

It was found that scale factors for the diagonal GG observations can be determined with an accuracy better than $4 \times$ $10^{-3}$. Concerning the common-mode accelerations, it seems feasible to estimate the associated biases with a high accuracy only for the $X$-direction (close to the along-track direction). A reliable estimate for the scale factors seems possible for the $Y$-axis (close to the cross-track direction) only (Visser 2007).

\subsection{Terrestrial gravity data}

Part of the external calibration process is performed as a comparison of calibrated GOCE GGs with GGs computed from terrestrial gravity data with the aim of having a strong indicator that the calibration has been successful. For this purpose four areas have been selected with high quality terrestrial gravity data. Three areas have been selected from gravitationally smooth regions (Arabelos and Tscherning 1998) with a wide global spatial distribution (Australia, Canadian Plains, Scandinavia) and a fourth area has been selected to examine a more gravitational "rough" area where more signal is left for the scale factor estimates (Norway). It is the intention that for each time the satellite passes a calibration area a trackwise comparison between GOCE measurements and the GGs computed from terrestrial data is done in the $\mathrm{MB}$ in terms of an estimate of a GG scale factor (SF), which for a successful evaluation should be close to 1 as the calibrated GGs from Sect. 5.1 are used as input.

The size of the areas has been chosen close to $12^{\circ} \times 12^{\circ}$ corresponding to the maximal distance flown in $200 \mathrm{~s}$, corresponding to $5 \mathrm{mHz}$, which is the lower bound of the MB. The area size and the spatial distribution ensures that GOCE will pass a calibration area approximately three times per day. With the calibration performed in the $\mathrm{MB}(5-100 \mathrm{mHz})$ the data should be filtered, and different filters were tested to extract the MB part of the gradients from the datasets, e.g. Chebyshev (types I and II) Butterworth, and elliptic 
filters. Also a filter combining subtraction of long wavelengths with a number of splines followed by Fourier analysis and subsequent band-pass filtering was tested. The results for different filters were in general quite similar, but the combined filter was chosen as baseline primarily because it was developed internally and therefore allows very easy access to configurable parameters such as the number of splines used and the band-pass width, and secondly it was preferred not to use filters already used previously in the calibration with global models (Butterworth filter). In the present procedure, GGs are calculated in all GOCE orbit points based on model data (EGM96) except in the calibration areas where the GGs are predicted from terrestrial gravity data by least-squares collocation. The terrestrial gravity data (gravity anomalies $\Delta \mathbf{g}_{i}$ in observation points $i$ ) are related to the anomalous gravity gradient $\mathbf{T}_{q r}$ as

$\Delta \mathbf{g}_{i}=L_{i}\left(\mathbf{T}_{q r}\right)+\mathbf{e}_{i}$

where $L_{i}\left(\mathbf{T}_{q r}\right)$ is the appropriate functional of the GG and $\mathbf{e}_{i}$ is the error of the observations (Tscherning 1976, 1993). The GG prediction in the GOCE orbit points $P$ is then calculated from

$\mathbf{T}_{q r}(P)=\mathbf{C}_{P i}^{T} \mathbf{C}_{i j}^{-1} \Delta \mathbf{g}_{j}$

where $\mathbf{C}_{i j}$ is the covariance matrix of the terrestrial observations and $\mathbf{C}_{P i}$ is a set of values of the covariance function (Moritz 1980). The two GG data sets, of which one consists of GG anomalies $\mathbf{T}_{q r}$ predicted from model/terrestrial data, the other of GG anomalies $\overline{\mathbf{T}}_{q r}$ from GOCE, are in the following treated equally.

Fourier analysis is used to extract the MB part of the gradients of both data sets. Because the signal is not periodic, it must be prepared before filtering by reducing the data for long wavelength distortion ( $1 / f$ errors) and noise. The signal is therefore split in a number of subsections, e.g. 300 for the present data set corresponding to intervals of approximately $5 \mathrm{~h}$. Splines are fitted to the intermediate periods and subtracted before the Fourier analysis. Fourier coefficients $F(k)$ for the data sets are determined by

$F(k)=\frac{1}{T_{N}} \int_{t=0}^{T_{N}} f(t) e^{-i k t} \mathrm{~d} t$

with $k=j \frac{2 \pi}{T_{N}}, T_{N}$ the measurement period and $N$ the number of observations $f(t)$. Coefficients $a_{j}$ and $b_{j}$ are extracted as

$F(k)=a_{j} / 2-i b_{j} / 2$, for $j=1,2, \ldots$

The equivalent function in the MB is then computed by summing the coefficients multiplied with cos or sin, respectively,

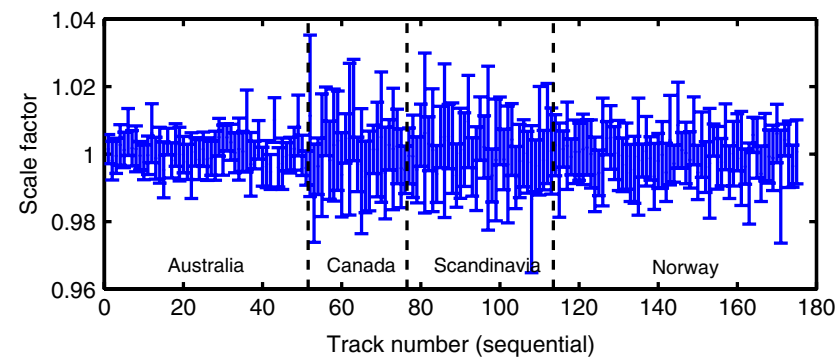

Fig. 11 Scale factors for the $V_{Z Z}$ gradient with error bars of $1 \sigma$ for the 4 areas using test data

to an integer $j$, corresponding to a wave-number equivalent to the frequencies of the MB. In complex notation

$\bar{T}_{q r}^{\mathrm{MB}}=\sum_{j=j_{1}}^{j_{2}} F\left(j \frac{2 \pi}{N}\right) e^{i \frac{2 \pi}{N} j}$.

The data sets are compared and scale factors and error estimates are determined for all gradients for each track passing a calibration area as

$T_{q r}^{\mathrm{MB}}(t)=s \cdot \bar{T}_{q r}^{\mathrm{MB}}(t)$

where $t$ is time and $s$ is scale factor. A least squares estimate of $s$ is

$s_{q r}=\frac{\sum_{t=\text { entry_time }}^{\text {exit_time }} T_{q r}^{\mathrm{MB}}(t) \cdot \bar{T}_{q r}^{\mathrm{MB}}(t)}{\sum_{t=\text { entry_time }}^{\text {exit_time }}\left(\bar{T}_{q r}^{\mathrm{MB}}(t)\right)^{2}}$

and the error estimate $\sigma_{s}^{2}$

$\sigma_{s}^{2}=\frac{\sum_{t=\text { entry_time }}^{\text {exit_time }} v^{2}}{\sum_{t=\text { entry_time }}^{\text {exit_time }}\left(\bar{T}_{q r}^{\mathrm{MB}}(t)\right)^{2}}$

where $v$ is the noise SD of the GGs. Tracks with only a few data points, e.g. near the corners of the areas, are discarded. The results for the $V_{Z Z}$ gradient are shown in Fig. 11 with error bars of $1 \sigma$. The SF estimates depend on the expected error of the gradients and in the presented results the error estimates are set to $10 \mathrm{mE}$ for all gradients. In Table 5, the SF mean, the SD and the maximum SF deviation from 1 are presented. The mean SF over all tracks in an area is 1 up to $10^{-3}$, while the maximum SF deviation from 1 is $10^{-2}$ for all three gradients, with $V_{Y Y}$ the more noisy gradient in this example.

The calibration method has changed during the HPF test phases from the original intention of bias, tilt and scale factor parameter estimation based on full GOCE GGs by collocation (Arabelos and Tscherning 1998; Bouman et al. 2004; Tscherning et al. 2006). However, as it was realised that the GOCE GG signal to noise ratio probably will make a collocation solution impossible, the method was changed to enable the extraction of data in the MB using filtering. Some of the 
Table 5 Statistics of scale factor estimates for diagonal GGs

\begin{tabular}{|c|c|c|c|c|c|c|c|c|c|}
\hline & \multicolumn{3}{|c|}{ SF mean } & \multicolumn{3}{|c|}{$\mathrm{SF} \sigma$ (mean of all tracks) } & \multicolumn{3}{|c|}{$\operatorname{Max}|\mathrm{SF}-1|$} \\
\hline & $V_{X X}$ & $V_{Y Y}$ & $V_{Z Z}$ & $V_{X X}$ & $V_{Y Y}$ & $V_{Z Z}$ & $V_{X X}$ & $V_{Y Y}$ & $V_{Z Z}$ \\
\hline Australia & 1.000 & 1.001 & 1.001 & 0.002 & 0.008 & 0.003 & 0.006 & 0.015 & 0.010 \\
\hline Canada & 1.001 & 1.000 & 1.000 & 0.006 & 0.008 & 0.006 & 0.012 & 0.018 & 0.013 \\
\hline Scandinavia & 1.001 & 1.000 & 1.000 & 0.007 & 0.013 & 0.005 & 0.016 & 0.029 & 0.013 \\
\hline Norway & 1.000 & 0.999 & 1.000 & 0.003 & 0.016 & 0.004 & 0.010 & 0.045 & 0.010 \\
\hline
\end{tabular}

Predicted error is $10 \mathrm{mE}$ for all gradients

advantages from the original method have not been carried over to the filtering method, e.g. it is not possible to estimate all parameters but only one parameter type (scale factor) and only for single tracks over a calibration area and not for a full area over time. In this way, the spatial correlation between the tracks is not utilised. Also the "old" method provided a means to estimate or validate biases of the attitude quaternions. However, with the high-frequency information from the terrestrial gravity data retained to a high degree in the predicted GGs the new method still provides valuable evaluation of GOCE GGs in the high frequency range, which may also effect the final choice of the frequency bound of the filter.

All in all, there are many switches that can be varied in this test, e.g., the GOCE gradient error estimates, the calibration area size, the MB limits, the data density and refinement of the spline interpolation technique, which all has a significant role to play in the SF estimates. Therefore, the calibration of the GGs with terrestrial data is not a "finished" package, but open for adjustment and fine tuning of these configurable parameters.

\subsection{Validation and discussion}

The calibration of the GOCE GG data using external gravity field information is a challenge, because GOCE is set out to provide a global gravity field model with unprecendented accuracy and resolution especially at medium to higher spatial resolutions. However, the GGs are not expected to be very accurate at long wavelengths, whereas GOCE SST data and existing global gravity field models do contain accurate long wavelength gravity field information. In addition, highly accurate terrestrial gravity data may regionally be used to calibrate the GOCE GGs at shorter wavelenghts. Before we briefly discuss and recapitulate our external calibration philosphy we go into the cross validation of the three calibration methods that have been implemented in the GOCE HPF and have been described in Sects. 5.1-5.3.

As said in Sect. 2, the calibration using global gravity field models is the baseline method. This method as well as the calibration using GOCE SST data may determine GG scale factors with an accuracy of $O\left(10^{-3}\right)$. Both methods use the signal power below the $\mathrm{MB}$ of the high-pass-filtered GG data, where specifically the method using GOCE SST data relies on an overlap in sensitivity of SST and GG observations to the Earth's gravity field. In the validation step, the $V_{X X}, V_{Y Y}$ and $V_{Z Z}$ scale factors of both methods will be compared and these should agree approximately up to the $O\left(10^{-3}\right)$ level. If so, this part of the validation has been successful, if not, more detailed analyses of the data are required. Beforehand, it is very difficult to predict how such analyses should be done. Obvious steps are to check whether the given calibration parameters really cover the same observation period, whether all input data has been correct, etc. More detailed analyses could include a recalibration using different calibration windows or alternative a priori global gravity field models. Also data from the calibration and monitoring facility (CMF) (González et al. 2007) or GOCE QL global gravity field models (Pail 2004) may contain hints should the validation have failed, that is, should the GG scale factor differences between the two calibration methods significantly exceed $10^{-3}$. However, as said above, this will be decided on a case-by-case basis including the increasing expert knowledge as more and more GOCE data will become available.

The GG data, which have been calibrated using global gravity field models, are input to the calibration using terrestrial gravity data. The GG scale factors as determined with this method should therefore be one, with an accuracy of approximately $10^{-2}$. Again, if this is the case, then this part of the validation has been successful. If not, then more detailed analysis of the data is required. Should this part of the validation fail, then analyses similar to the previous case are required with special focus on the $\mathrm{MB}$, because there lies the strength of the terrestrial gravity data.

One definition of calibration ${ }^{2}$ is that it is the process of establishing the relationship between a measuring device and the units of measure, which is done by comparing a device or the output of an instrument to a standard having known measurement characteristics. This is exactly the purpose of

\footnotetext{
${ }^{2}$ http://en.wikipedia.org/wiki/Calibration.
} 
all three calibration methods discussed: scale factors and possibly other calibration parameters are determined for the different GGs and/or the common-mode accelerations. A valid question with respect to our calibration methods could be whether they are needed at all as they seem not to be able, at least in the given example, to improve upon the a priori GOCE GG scale factors (assumed to be one after in-flight calibration). Furthermore, GOCE data are being used in our external calibration methods and it is therefore the question how external is defined in our approach.

To answer the first question, that is, what is the added value of the three presented external calibration methods with respect to the GOCE in-flight calibration method, we need to summarize a few details of the in-flight calibration and the GOCE data processing in general. The in-flight calibration method uses random shaking applied about each axis by the gradiometer calibration device (cold-gas thrusters) and the ion thruster (Cesare and Catastini 2005). The satellite shaking data are being used together with star sensor data to determine scale factors, couplings and misalignments of the common and differential accelerations. The whole procedure as described in (Cesare and Catastini 2005) is quite involved and employs empirical relationships between accelerometer common scale factors and relies on the assumption that the GG signal in the upper measurement band, $0.05-0.1 \mathrm{~Hz}$, is negligible. The in-flight-calibrated differential accelerations are then used to compute angular accelerations. The angular accelerations are numerically integrated to obtain angular rates, which are combined with star sensor quaternions using a Kalman filter. A cut-off frequency is used below the measurement band, where the $1 / f$ acceleration error starts to dominate the star sensor error. Finally, the GGs are computed by subtracting the proper combinations of angular rates from the differential accelerations. For a comprehensive description of the above steps (see Cesare and Sechi 2005).

Altogether, the in-flight calibration and gradiometer data processing is complex and for example, acceleration common scale factors, which have been determined by in-flight calibration, do not directly propagate to GG scale factors. In addition, a gradiometer in space has never been flown before and it has been built with state-of-the-art technologies. It is therefore essential also to have calibration methods available that directly operate on the GGs as opposed to accelerations in the in-flight calibration, for GGs are the quantities from which an accurate GOCE gravity field model is to be derived.

At first sight, it seems to be contradictory to calibrate the GOCE GGs using an existing global gravity field model, since, after all, the GOCE gravity field solution should have unprecedented accuracy and resolution. At long wavelengths, the GOCE GG data are less accurate, but the signal-to-noise ratio may still be much larger than 1 (see Fig. 2). Existing state-of-the-art global gravity field models have a high precision at long wavelengths, which thus allows a comparison with the GOCE GGs. Especially the signal at one and two CPR, well below the measurement band, is strong and it is the signal at these frequencies that will dominate the calibration. It may therefore not be very important whether we use a satellite-only or a combined global gravity field model as long as the total power for frequencies below the measurement band is correct. Typically, the maximum spherical harmonic degree of satellite-only models is less than that of combined models, while usually also some sort of regularisation is applied. Both the regularisation and the lower maximum degree may lead to powerloss, which is why we have chosen to use a combined model to calibrate with. Of course, these models contain terrestrial gravity data and/or satellite altimetry data, which in many cases will necessarily be poorer than what GOCE will provide. However, we repeat again that the calibration using global gravity field models will mainly rely on the signal at one and two CPR. In addition, the model GGs we are calibrating have global coverage and errors may average out. Furthermore, systematic errors such as dynamic ocean topography may be neglible, because we use GGs, that is, the second order spatial derivative. All in all, we think that is feasible to use existing global gravity field models for GG calibration.

Because the calibration of the novel gradiometer instrument is a delicate topic, a number of cross-checks has been implemented. First of all, after the calibration using global gravity field models, the trace SD in the measurement band is checked and compared with that of the not externally calibrated original GOCE GGs (as described in Sect. 5.1). Secondly, two independent external calibration methods are used to validate the global model calibration results, one using GOCE SST data and the other using terrestrial gravity data. Although the SF accuracy that can be achieved with the method using terrestrial gravity data may be somewhat less as compared with the other two methods, this method is essential as it is the only external calibration method with which we are able to determine GG scale factors directly in the measurement band.

We will close with a few words on how we define "external" in our external calibration methods. With external data we mean data external to the GOCE gradiometer, not necessarily external to the GOCE satellite: the GOCE SST data is data independent of the gradiometer and therefore external, while GOCE orbit and star sensor data are needed to compute GG calibration values from either a global gravity field model or terrestrial gravity data. In this context, the use of star sensor or gradiometer quaternions deserves attention. On the one hand, the star sensor quaternions, differentiated to obtain angular rates, are used in the in-flight calibration to determine accelerometer scale factors. These quaternions are also needed to relate the GRF to an inertial or Earth-fixed frame. So, in a sense, these data are used twice. However, while the differentiated star sensor quaternions are used as 
standard in the in-flight calibration, they provide reference frame information in the external calibration, and in this case, the external gravity information is used as standard to calibrate with. The gradiometer quaternions, on the other hand, combine star sensor quaternions at low frequencies and gradiometer data for higher frequencies. The use of these quaternions in the external calibration seems undesirable, because gradiometer data are applied in the calibration of gradiometer data. The situation is somewhat subtle, however. The gradiometer data that go into the quaternions are not used as such, but they are integrated twice. Hence, the original accelerometer error, approximately flat in the MB, will be suppressed for higher frequencies and will be (far) below the star sensor quaternion error that exhibits white noise for higher frequencies. Also remaining errors in the accelerometer data, for example due to imperfect in-flight calibration, will be reduced by the integration process as far as the quaternions is concerned. To summarise, the gradiometer quaternions consist mainly of star sensor information at low frequencies while they have an improved performance at higher frequencies as compared with star sensor quaternions. In any case, the difference may be small between model GGs generated using star sensor or gradiometer quaternions. For example, if we use the simulated data of the first week and generate two sets of $V_{X X}$ model GGs using either star sensor or gradiometer quaternions, then the power spectral density of the relative differences between the two sets is generally below $10^{-4}$ for all frequencies. In the MB, the PSD of the relative differences is below $10^{-5}$ and flat, which can be attributed to the star sensor white noise for higher frequencies.

\section{Conclusions}

The preprocessing of the GOCE GGs at the GOCE highlevel processing facility entails corrections for temporal gravity field variations, outlier detection, external calibration and error assessment. The temporal GG corrections are generally below the GG error level, which is predicted to show a $1 / f$ behaviour for low frequencies. However, the temporal signals may be systematic, and to be consistent with the GOCE SST processing, the GOCE GGs are corrected for the temporal effects. The $1 / f$ error on the GOCE GGs, as well as the reconstruction of the reference frame in which the GGs are given, may pose a challenge to the GG preprocessing. A direct comparison, for example, with model gradients is not possible and the $1 / f$ error needs to be taken into account. In the outlier detection, a moving window is used in which the local median is subtracted. Subsequently, the GGs are compared with model gradients and the trace condition is used in the outlier detection for the diagonal GGs. The local median acts as a high-pass filter and the use of the median improves the robustness of the method. For the GG calibra- tion global gravity field models, GOCE GPS data as well as terrestrial gravity data are used. All three methods use a high-pass filter to suppress the $1 / f$ GG error. Both the GOCE GGs as well as the model GGs are filtered before the actual calibration step. The GG scale factors can be determined up to the $O\left(10^{-2}\right)$ level in the MB using terrestrial gravity data and up to the $O\left(10^{-3}\right)$ level for the other two methods. Best calibration results are obtained for the baseline method using global gravity field models if instrument misalignments are estimated in addition to the GG scale factors.

Acknowledgments This study was performed in the framework of the European Space Agency project (No. 18308/04/NL/MM): GOCE High-level Processing Facility.

Open Access This article is distributed under the terms of the Creative Commons Attribution Noncommercial License which permits any noncommercial use, distribution, and reproduction in any medium, provided the original author(s) and source are credited.

\section{References}

Abrikosov O, Jarecki F, Müller J, Petrovic S, Schwintzer P (2006) The impact of temporal gravity variations on GOCE gravity field recovery. In: Flury J, Rummel R, Reigber C, Rothacher M, Boedecker G, Schreiber U (eds) Observation of the Earh system from space. Springer, Heidelberg, pp 255-269

Albertella A, Migliaccio F, Sansò F, Tscherning C (2000) The spacewise approach—overall scientific data strategy. In: Sünkel H (ed) From Eötvös to mGal, Final report. ESA/ESTEC contract no. 13392/98/NL/GD

Arabelos D, Tscherning C (1998) Calibration of satellite gradiometer data aided by ground gravity data. J Geod 72:617-625

Bouman J (2004) Quick-look outlier detection for GOCE gravity gradients. Newton's Bull 2:78-87

Bouman J, Koop R (2003a) Calibration of GOCE SGG data combining terrestrial gravity data and global gravity field models. In: Tziavos I (ed) Gravity and geoid 2002; 3rd meeting of the IGGC Ziti editions, pp 275-280

Bouman J, Koop R (2003b) Error assessment of GOCE SGG data using along track interpolation. Adv Geosci 1:27-32

Bouman J, Koop R, Tscherning C, Visser P (2004) Calibration of GOCE SGG data using high-low SST, terrestrial gravity data, and global gravity field models. J Geod 78:124-137

Bouman J, Kern M, Koop R, Pail R, Haagmans R, Preimesberger T (2005) Comparison of outlier detection algorithms for GOCE gravity gradients. In: Jekeli C, Bastos L, Fernandes J (eds) Gravity, geoid and space missions, vol 129, International Association of Geodesy Symposia. Springer, Heidelberg, pp 83-88

Bouman J, Rispens S, Koop R (2007) GOCE gravity gradients for use in earth sciences. In: Proceedings of the 3rd international GOCE user workshop. ESA-ESRIN, Frascati, Italy, 6-8 November 2006, ESA SP-627

Catastini G, Cesare S, Sanctis SD, Dumontel M, Parisch M, Sechi G (2007) Predictions of the GOCE in-flight performances with the end-to-end system simulator. In: Proceedings of the 3rd international GOCE user workshop. ESA-ESRIN, Frascati, Italy, 6-8 November 2006, ESA SP-627

Cesare S (2005) Performance requirements and budgets for the gradiometric mission. Issue 3 GO-TN-AI-0027, Alenia Spazio, Turin 
Cesare S, Catastini G (2005) Gradiometer on-orbit calibration procedure analysis. Issue 3 GO-TN-AI-0069, Alenia Spazio, Turin

Cesare S, Sechi G (2005) Gradiometer ground processing algorithms documentation. Issue 6 GO-TN-AI-0067, Alenia Spazio, Turin

Dobslaw H, Thomas M (2007) Simulation and observation of global ocean mass anomalies. J Geophys Res 112:(C05040). doi:10.1029/ 2006JC004035

Drijfhout S, Heinze C, Latif M, Maier-Reimer E (1996) Mean circulation and internal variability in an ocean primitive equation model. J Phys Oceanogr 26:559-580

ECMWF (2007). IFS documentation. CY31r1 operational implementation, Part 1-7. http://www.ecmwf.int/research/ifsdocs/CY31r1/ index.html

ESA (1999) Gravity field and steady-state ocean circulation mission. Reports for mission selection; the four candidate earth explorer core missions. ESA SP-1233(1)

Eshagh M, Sjöberg L (2008) Topographic and atmospheric efects of GOCE gradiometric data in a local north-oriented frame: a case study in Fennoscandia and Iran. Studia Geophysica et Geodaetica (accepted)

Farrell W (1972) Deformation of the earth by surface loads. Rev Geophys Space Phys 10:761-797

Flechtner F (2007) AOD1B product description document for product release 01 to 04. Document GRACE, issue 3.1, pp 327-750

Foerste C, Schmidt R, Stubenvoll R, Flechtner F, Meyer U, Koenig $\mathrm{R}$, Neumayer H, Biancale R, Lemoine J-M, Bruinsma S, Loyer S, Barthelmes F, Esselborn S (2007) The GeoForschungsZentrum Potsdam/Groupe de Recherche de Géodésie Spatiale satellite-only and combined gravity field models: EIGEN-GL04S1 and EIGENGL04C. J Geod. doi:10.1007/s00190-007-0183-8

González J, Canales A, Acarreta J, López-Hazas E, de Candia D, Salzo S, Floberghagen R (2007) The GOCE calibration and monitoring facility (CMF). In: Proceedings of the 3rd international GOCE user workshop. ESA-ESRIN, Frascati, Italy, 6-8 November 2006, ESA SP-627

Gruber T, Rummel R (2006) Concept and capability of GOCE. In: Proceedings of the GOCINA workshop, vol 25. Cahiers du Centre Europeen de Geodynamique et de Seismologie, pp 31-37

Gruber T, Rummel R, Abrikosov O, van Hees R (2007a) GOCE level 2 product data handbook. GO-MA-HPF-GS-0110, issue 3.3

Gruber T, Rummel R, Koop R (2007b) How to use GOCE level 2 products. In: Proceedings of the 3rd international GOCE user workshop. ESA-ESRIN, Frascati, Italy, 6-8 November 2006, ESA SP-627

Hoaglin D, Mosteller F, Tukey J (1983) Understanding robust and exploratory data analysis. Wiley, New York

IERS Conventions (2004) IERS technical note no. 32. Technical report, Verlag des Bundesamtes für Kartographie und Geodäsie, Frankfurt am Main. Available at www.iers.org/iers/publications/tn/tn32/ tn32.pdf

Jarecki F, Müller J (2007) GOCE gradiometer validation in satellite track cross-overs. In: Kilicoglu A, Forsberg R (eds) Gravity field of the Earth, vol 73. of Harita Dergisi. Harita Genel Komutanligi, Ankara. Proceedings of the 1st international symposium of the gravity field service, pp 223-228

Jarecki F, Wolf K, Denker H, Müller J (2006) Quality assessment of GOCE gradients. In: Flury J, Rummel R, Reigber C, Rothacher M, Boedecker G, Schreiber U (eds) Observation of the Earh System from Space. Springer, Heidelberg, pp 271-285

Kern M, Preimesberger T, Allesch M, Pail R, Bouman J, Koop $\mathrm{R}$ (2005) Outlier detection algorithms and their performance in GOCE gravity field processing. J Geod 78:509-519

Koop R, Bouman J, Schrama E, Visser P (2002) Calibration and error assessment of GOCE data. In: Ádám J, Schwarz K-P (eds) Vistas for geodesy in the new millenium, vol 125, International
Association of Geodesy Symposia. Springer, Heidelberg, pp $167-$ 174

Koop R, Gruber T, Rummel R (2007) The status of the GOCE high-level processing facility. In: Proceedings of the 3rd international GOCE user workshop. ESA-ESRIN, Frascati, Italy, 6-8 November 2006, ESA SP-627

Lambeck K (1988) Geophysical geodesy, the slow deformations of the earth. Clarendon Press, Oxford

Lemoine F, Kenyon S, Factor J, Trimmer R, Pavlis N, Chinn D, Cox C, Klosko S, Luthcke S, Torrence M, Wang Y, Williamson R, Pavlis E, Rapp R, Olson T (1998) The development of the joint NASA GSFC and the National Imagery and Mapping Agency (NIMA) geopotential model EGM96. TP 1998-206861, NASA Goddard Space Flight Center

Li Y (2001) 3-D inversion of gravity gradiometer data. Electrical and magnetic studies, Department Geophysics, Colorado School of Mines

Migliaccio F, Reguzzoni M, Sansò F (2004) Space-wise approach to satellite gravity field determination in the presence of coloured noise. J Geod 78:304-313

Moritz H (1980) Advanced physical geodesy. Wichmann, Karlsruhe

Overhauser A (1968) Analytic definition of curves and surfaces by parabolic blending. Techn. report no. SL68-40, Scientific Research Staff Publication, Ford Motor Company, Detroit

Pail R (2004) GOCE quick-look gravity field analysis: treatment of gravity gradients defined in the gradiometer reference frame. In: Proceedings of the 2nd international GOCE user workshop. ESAESRIN, Frascati, Italy, 8-10 March 2004, ESA SP-569

Pail R (2005) A parametric study on the impact of satellite attitude errors on GOCE gravity field recovery. J Geod 79:231-241

Pail R, Plank G (2004) Gravity field processing strategy. Stud Geophys Geod 48:289-309

Pawlowski B (1998) Gravity gradiometry in resource exploration. Leading Edge 17:51-52

Rowlands D, Marshall J, McCarthy J, Moore D, Pavlis D, Rowton S, Luthcke S, Tsaoussi L (1995) Geodyn ii system description. Contractor report vols 1-5. Hughes STX Corp., Greenbelt, MD

Schmidt R, Schwintzer P, Flechtner F, Reigber C, Gütner A, Döll P, Ramillien G, Cazenave A, Petrovic S, Jochmann H, Wünsch J (2006) GRACE observations of changes in continental water storage. Glob Planet Change 50:112-126

Schrama E (1995) Gravity Research Missions reviewed in the light of the indirect ocean tide potential. In: Rapp R, Cazenave A, Nerem $\mathrm{R}$ (eds) Global gravity field and its temporal variations, vol 116, International Association of Geodesy Symposia. Springer, Heidelberg, pp 131-140

Schrama E, Ray R (1994) A preliminary tidal analysis of Topex/Poseidon altimetry. J Geophys Res 99(C12):2479924808

Standish E (1998) JPL planetary and lunar ephemerides DE405/LE405. Technical report JPL IOM 312.F - 98 - 047, JPL Pasadena CA

Tapley B, Ries J, Bettadpur S, Chambers D, Cheng M, Condi F, Gunter B, Kang Z, Nagel P, Pastor R, Pekker T, Poole S, Wang F (2005) GGM02 - an improved Earth gravity field model from GRACE. J Geod 79(8):467-478. doi:10.1007/s00190-005-0480-z

Teunissen P (2000) Testing theory; an introduction. Delft University Press, Delft

Thomas M (2002) Ocean induced variations of the Earth's rotationresults from a simultaneous model of global circulation and tides. $\mathrm{Ph} . \mathrm{D}$. thesis, University of Hamburg, Germany

Tscherning C (1976) Covariance expressions for second and lower order derivatives of the anomalous potential. Report no. 225, Department of Geodetic Science and Surveying, Ohio State University 
Tscherning C (1993) Computation of covariances of derivatives of the anomalous gravity potential in a rotated reference frame. Manusc Geod 8(3):115-123

Tscherning C, Veicherts M, Arabelos D (2006) Calibration of GOCE gravity gradient data using smooth ground gravity. In: Proceedings of the GOCINA workshop, vol 25. Cahiers du Centre Europeen de Geodynamique et de Seismologie, pp 63-67

Velicogna I, Wahr J (2006) Measurements of time-variable gravity snow mass loss in Antarctica. Science 311:1754-1756

Visser P (2007) GOCE gradiometer validation by GPS. Adv Space Res 39(10):1630-1637. doi:10.1016/j.asr.2006.09.014

Visser P, van den IJssel J (2000) GPS-based precise orbit determination of the very low Earth orbiting gravity mission GOCE. J Geod 74(7/8):590-602

Visser P, van den IJssel J, Koop R, Klees R (2001) Exploring gravity field determination from orbit perturbations of the European Gravity Mission GOCE. J Geod 75(2/3):89-98
Visser P, van den IJssel J, van Helleputte T, Bock H, Jaeggi A, Beutler G, Hugentobler U, Svehla D (2007) Rapid and precise orbit determination for the GOCE Satellite. In: Proceedings of the 3rd international GOCE user workshop. ESA-ESRIN, Frascati, Italy, 6-8 November 2006, ESA SP-627

Wolff J, Maier-Reiner E, Legutke S (1996) The Hamburg ocean primitive equation model hope. Technical report no. 13, DKRZ, Hamburg

Yunck T, Bertiger W, Wu S, Bar-Sever Y, Christensen E, Haines B, Lichten S, Muellerschoen R, Vigue Y, Willis P (1994) First assessment of GPS-based reduced dynamic orbit determination on TOPEX/Poseidon. Geophys Res Lett 21(7):541-544 